\title{
Optimal Time to Sell in Real Estate Portfolio Management
}

\author{
Fabrice Barthélémy and Jean-Luc Prigent \\ ThEMA, University of Cergy-Pontoise, Cergy-Pontoise, France \\ E-mails: fabrice.barthelemy@u-cergy.fr; jean-luc.prigent@u-cergy.fr
}

\begin{abstract}
This paper examines the properties of optimal times to sell a diversified real estate portfolio. The portfolio value is supposed to be the sum of the discounted free cash flows and the discounted terminal value (the discounted selling price). According to Baroni et al. (2007b), we assume that the terminal value corresponds to the real estate index. The optimization problem corresponds to the maximization of a quasi-linear utility function. We consider three cases. The first one assumes that the investor knows the probability distribution of the real estate index. However, at the initial time, he has to choose one deterministic optimal time to sell. The second one considers an investor who is perfectly informed about the market dynamics. Whatever the random event that generates the path, he knows the entire path from the beginning. Then, given the realization of the random variable, the path is deterministic for this investor. Therefore, at the initial time, he can determine the optimal time to sell for each path of the index. Finally, the last case is devoted to the analysis of the intertemporal optimization, based on the American option approach. We compute the optimal solution for each of these three cases and compare their properties. The comparison is also made with the buy-and-hold strategy.
\end{abstract}

Key Words Real estate portfolio, Optimal holding period, American option.

JEL Classification C61, G11, R21. 


\section{Introduction}

The research concerning real estate holding period is rather limited and exclusively empirical. For the US, the holding durations are mainly determined by tax laws as shown by Hendershott and Ling (1984), Gau and Wang (1994) or Fisher and Young (2000). For small residential investment, Brown and Geurts (2005) examine empirically how long does an investor own an apartment building. They found that the average holding period is around five years, through a sample of apartment buildings of between 5 and 20 units over the period 1970-1990 in the city of San Diego. For the UK market, Rowley, Gibson and Ward (1996) emphasized the existence of ex ante expectations about holding periods, for real real estate investors or new property developers. Indeed, the holding period decision is related to depreciation or obsolescence factors. Brown (2004) proves that the risk peculiar to real estate investments may explain the behaviour of real estate investors. However, applying the CAPM for individuals to understand their portfolio management does not yield relevant results, as shown by Geltner and Miller (2001).

Collett, Lizieri and Ward (2003) examine empirically the ex post holding periods and show that these values are higher than those claimed by investors. Knowing the holding period is important for investment in commercial real estate portfolios and moreover the analysis period has to be specified. Using the database of properties provided by IPD in the UK over an 18-year period, their empirical analysis shows that the median holding period is about seven years. Additionally, higher the return, lower the holding period. However, this empirical study cannot lead to conclusions about the relation between asset volatility and holding period. This is due to the absence of proxy to measure this eventual relationship.

In this paper, we search for theoretical optimal times to sell in a real estate portfolio. As a byproduct, our models provide analytical solutions whose properties illustrate most of the previous empirical results. For this purpose, we extend the model of Baroni et al. (2007a, b) by considering two other maximization issues. ${ }^{1}$ First, we determine the optimal holding period if the investor is perfectly informed about the growth rate dynamics. This corresponds to the best ideal case where the investor, as a new period starts, would know exactly the price dynamics, and would be able to choose the best time to sell the asset. This approach determines the upper bound of the present value of the portfolio as a function of holding period policy, in the sense that it maximizes present value using perfect forsight. This case thus serves as a sort of benchmark. In Baroni et al. (2007b), the investor does not know the dynamics, but only knows its probability distribution. The solutions are analyzed by using simulations and quasi explicit formulae. Secondly, we study the optimal holding period for an intertemporal maximization, according to the American option approach. In that case, at each time during a given management period, the investor compares the present portfolio value (the discounted value of the selling price at this time) with the maximal expected value he could have if he would keep the asset. He sells as soon as the present value is higher than this expectation.

This is a completely different issue than the random times in a real estate portfolio context examined by Bond et al. (2007). In their model, the marketing period risk corresponds to time-on-the-market that indicates how long it takes to sell an asset once you put it on the market to sell it. It is an exogenous random variable. In our model, the times to sell are endogenously determined from the optimization problems. 
The structure of the paper is laid out as follows: Section 2 presents results of Baroni et al. (2007b) in a continuous-time framework. Section 3 provides the analysis of the perfectly informed investor. Section 4 develops the American option approach. Comparisons of these three approaches are presented in Section 5. In particular, we emphasize the case for which the deterministic optimal time is not degenerated (neither equal to the initial time nor to the maturity). Moreover, in this section, we also introduce the buy-and hold strategy. Most of the proofs are gathered in the Appendix.

\section{Optimal time $T *$ to sell, chosen at time 0}

In this section, the time of sale is pre-set, committed irrevocably at time 0 , based on the expected dynamics of the portfolio value and its cash flow. The real estate portfolio value is defined as the sum of the discounted free cash flows (FCF) and the discounted terminal value (the selling price). Denote $k$ as the weighted average cost of capital (WACC), which is used to discount the different free cash flows, and the terminal value. We assume that the free cash flow grows at a constant rate $g{ }^{2}$

\subsection{Continuous-time model}

As Baroni et al. (2007a), we suppose that the price dynamics, which corresponds to the terminal value of a diversified portfolio (for instance a real estate index), follows a geometric Brownian motion:

$$
\frac{d \widetilde{P}_{t}}{\widetilde{P}_{t}}=\mu d t+\sigma d W_{t},
$$

where $W_{t}$ is a standard Brownian motion. We have:

$$
\tilde{P}_{t}=P_{0} \exp \left[\left(\mu-1 / 2 \sigma^{2}\right) t+\sigma W_{t}\right] .
$$

This equation assumes that the real estate return can be modelled as a simple diffusion process where parameters $\mu$ and $\sigma$ are respectively equal to the trend and to the volatility. The expected return of the asset at time $t$ is given by:

$$
E\left[\frac{\widetilde{P}_{t}}{P_{0}}\right]=\exp (\mu t) .
$$

Then the future real estate index value at time $t$, discounted at time 0 , can be expressed as:

$$
P_{t}=P_{0} \exp \left[\left(\mu-k-1 / 2 \sigma^{2}\right) t+\sigma W_{t}\right]
$$

with

$$
E\left[\frac{P_{t}}{P_{0}}\right]=\exp ([\mu-k] t) .
$$

Denote by $F C F_{0}$ the initial value of the free cash flow. The continuous-time version of the sum of the discounted free cash flows $F C F_{s}$ is equal to:

which leads to

$$
C_{t}=\int_{0}^{t} F C F_{s} e^{-k s} d s=\int_{0}^{t} F C F_{0} e^{-[k-g] s} d s,
$$




$$
C_{t}=\frac{F C F_{0}}{k-g}\left(1-e^{-[k-g] t}\right)
$$

Introduce the real estate portfolio value process $V$, which is the sum of the discounted free cash flows and the future real estate index value at time $t$, discounted at time 0:

$$
V_{t}=C_{t}+P_{t}
$$

\subsection{Characteristics of $V_{\bar{T}}$}

We determine the portfolio value $V_{\bar{T}}$ for a given maturity $\bar{T}$. This assumption on the time horizon allows to take account of selling constraints before a limit date. The higher $\bar{T}$, the less stringent this limit. Additionally, this hypothesis allows the study of buy-and-hold strategies (see section 5). The future portfolio value at maturity, discounted at time 0 , is given by:

$$
V_{\bar{T}}=\frac{F C F_{0}}{k-g}\left(1-e^{-[k-g] \bar{T}}\right)+P_{0} \exp \left[\left(\mu-k-1 / 2 \sigma^{2}\right) \bar{T}+\sigma W_{\bar{T}}\right] .
$$

The portfolio value $V_{\bar{T}}$ is the sum of a deterministic component and a Lognormal random variable.

\subsection{Determination of $T^{*}$}

We determine the optimal solution at time 0 , for a given maturity $\bar{T}$ and for an investor maximising a quasi linear expected utility. First note that the sum of the discounted free cash flows $\left(C_{t}\right)$ is always increasing due to the cash accumulation over time. Second, we have to analyze the expectation of the future real estate index value at time $t$, discounted at time 0 : if the price return $\mu$ is higher than the WACC $k$, then, the optimal solution is simply equal to the maturity $\bar{T}$. Thus, in what follows, we consider the case $\mu<k$. Consequently, not selling the asset implies a higher but a smaller discounted expected terminal value $P_{0} e^{[\mu-k] t}$. Hence, the investor has to balance between more (discounted) flows and less expected discounted index value.

We also focus on the sub case $g<\mu$, which corresponds to empirical data ${ }^{3}$.

The optimization problem is:

$$
\operatorname{Max}_{t \in[0, \bar{T}]} E\left[V_{t}\right]
$$

Since the expectation of $V_{t}$ is equal to:

$$
E\left[V_{t}\right]=\frac{F C F_{0}}{k-g}\left(1-e^{-[k-g] t}\right)+P_{0} e^{[\mu-k] t},
$$

we deduce:

$$
\frac{\partial E\left[V_{t}\right]}{\partial t}=F C F_{0} e^{-[k-g] t}+P_{0}(\mu-k) e^{[\mu-k] t}
$$


Then, the optimal holding period is determined as follows.

Case 1: The initial price $P_{0}$ is smaller than $\frac{F C F_{0}}{k-\mu} e^{-(k-\mu) \bar{T}}$.

Then, the optimal time to sell $T^{*}$ corresponds to the maturity $\bar{T}$. Since the Price Earning Ratio (PER) $\frac{P_{0}}{F C F_{0}}$ is too small $\left(<\frac{e^{-(k-\mu) \bar{T}}}{k-\mu}\right)$, the sell is not relevant before maturity.

Case 2: The initial price $P_{0}$ lies between the two values $\frac{F C F_{0}}{k-\mu} e^{-(k-\mu) \bar{T}}$ and $\frac{F C F_{0}}{k-\mu}$.

Then, the optimal time to sell $T^{*}$ is solution of the following equation:

$$
\frac{\partial E\left[V_{t}\right]}{\partial t}=0
$$

From Equation (11), we deduce ${ }^{4}$ :

$$
T^{*}=\frac{1}{\mu-g} \ln \left(\frac{F C F_{0}}{P_{0}} \times \frac{1}{k-\mu}\right) .
$$

In particular, note that $T^{*}$ is a decreasing function of the initial price $P_{0}$ and of the difference between the index return $\mu$ and the growth rate $g$ of the free cash flows. This latter property was empirically observed by Brown and Geurts (2005). It means that investors sell property sooner when values rise faster than rent.

Case 3: The initial price $P_{0}$ is higher than $\frac{F C F_{0}}{k-\mu}$.

Then, the optimal time to sell $T^{*}$ corresponds to the initial time 0 . Since the PER $\frac{P_{0}}{F C F_{0}}$ is sufficiently large $\left(>\frac{1}{k-\mu}\right)$, there is no reason to keep the asset $P$. As an illustration, the cumulative value $C_{t}$ of the $F C F_{t}$ values, of the expectation of the index value $E\left[P_{t}\right]$ and the expectation of the portfolio value $E\left[V_{t}\right]$ are displayed in Figure 1. We consider two sets of parameter values for a 20 year management period $(\bar{T}=20)$.

We note that the discounted expected value $V_{t}$ of the portfolio is concave. The parameter values imply that the optimal holding period, $T^{*}$, is respectively equal to 9.13 years and 16.11 years. For these two examples, the optimal time to sell $T^{*}$ is smaller than the maturity $\bar{T}$. In the second example, the discounted portfolio value varies up to $20 \%{ }^{5}$

Knowing the optimal time to sell $T^{*}$, which is deterministic, the probability distribution of the discounted portfolio value $V_{T^{*}}$ can be determined. The value $V_{T^{*}}$ is equal to:

$$
V_{T^{*}}=\frac{F C F_{0}}{(k-g)}\left(1-e^{-(k-g) T^{*}}\right)+P_{0} \exp \left[\left(\mu-k-1 / 2 \sigma^{2}\right) T^{*}+\sigma W_{T^{*}}\right] .
$$

Denote $A=\frac{F C F_{0}}{(k-g)}\left(1-e^{-(k-g) T^{*}}\right)$ the cumulative discounted free cash flow value at $T^{*}$. Since, from (13), the optimal time to sell satisfies: 


$$
T^{*}=\frac{1}{\mu-g} \ln \left(\frac{F C F_{0}}{P_{0}(k-\mu)}\right),
$$

then, we deduce:

$$
A=\frac{F C F_{0}}{(k-g)}\left(1-\left[\frac{F C F_{0}}{P_{0}(k-\mu)}\right]^{\left(-\frac{k-g}{\mu-g}\right)}\right),
$$

and the cdf $F_{V_{T^{*}}}$ of $V_{T^{*}}$ is given by:

$$
F_{V_{T^{*}}}(v)=\left\{\begin{array}{c}
0, \text { if } v \leq A \\
N\left[\frac{1}{\sigma \sqrt{T^{*}}}\left(\ln \left(\frac{v-A}{P_{0}}\right)-\left(\left(\mu-k-1 / 2 \sigma^{2}\right) T^{*}\right)\right)\right] \text {, if } v>A
\end{array}\right.
$$

where $N$ denotes the cdf of the standard Gaussian distribution.

Fig. $1 C_{t}, E\left[P_{t}\right]$ and $E\left[V_{t}\right]$ as functions of time $t$ in $[0,20]$
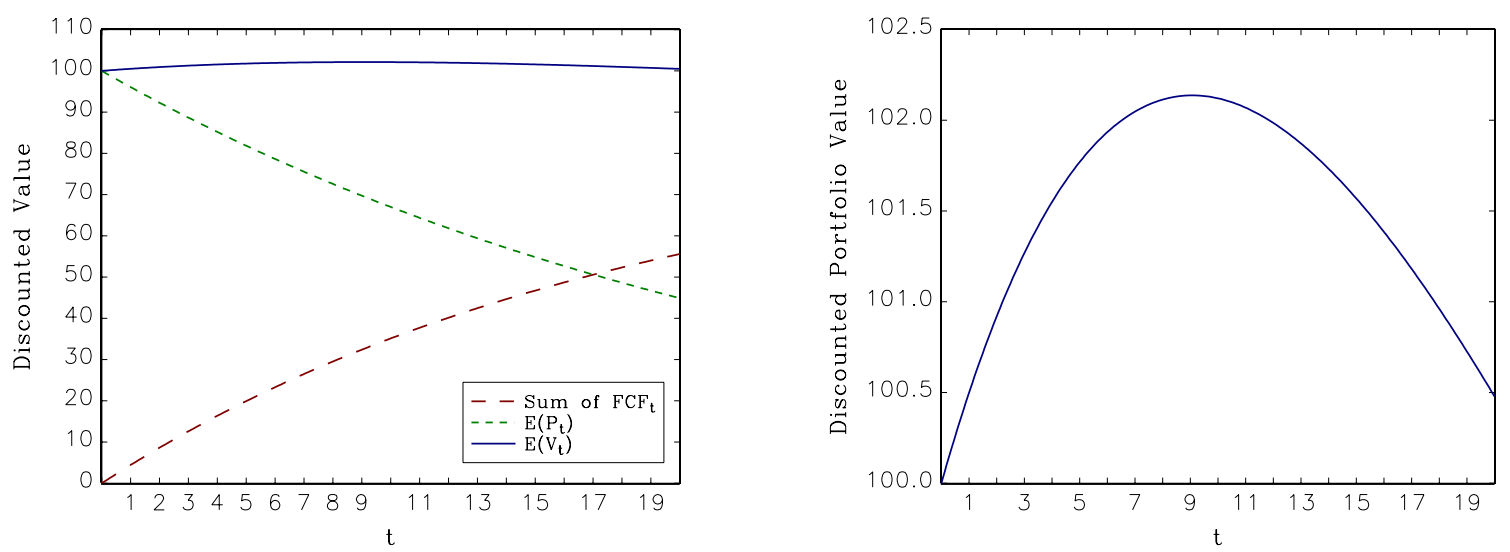

Case 1: $\mu=4.4 \%, g=3 \%, k=8.4 \%, P_{0}=100, F C F_{0}=100 / 22$.
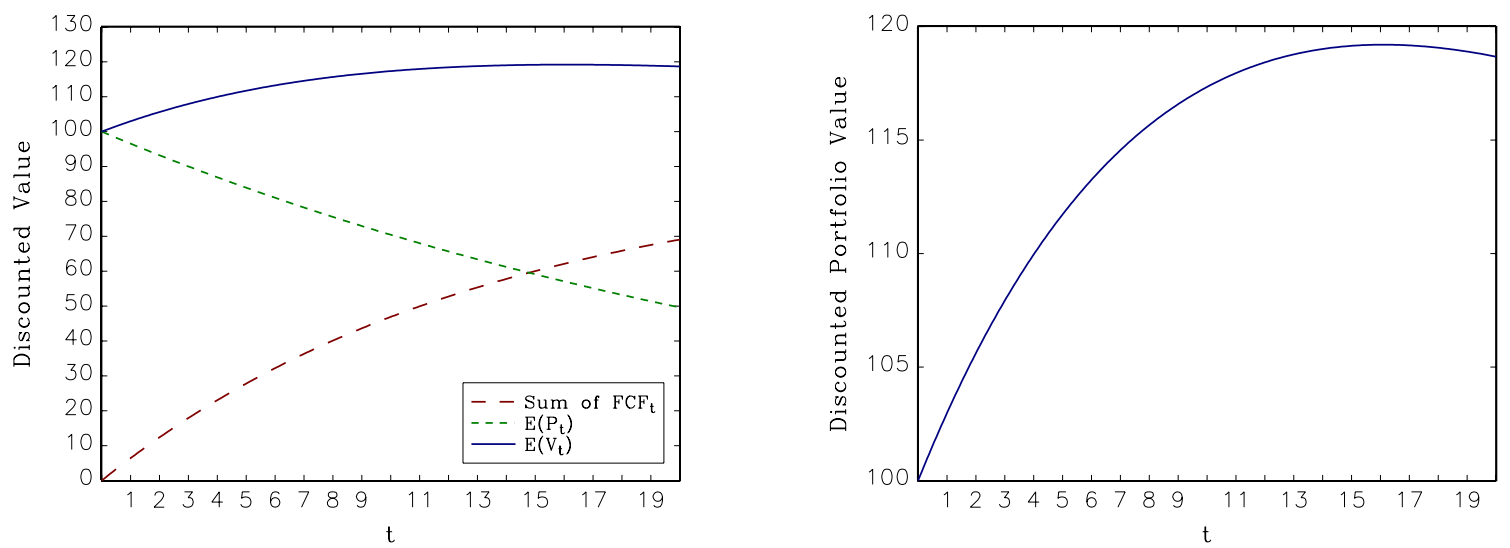

Case 2: $\mu=6 \%, g=2 \%, k=9.5 \%, P_{0}=100, F C F_{0}=100 / 15$.

The pdf of $V_{T^{*}}$ are illustrated in Figure 2 for two different volatilities. Higher the volatility, larger the range of admissible discounted portfolio values. Up to a translation, these are the pdfs of a Lognormal distribution, with a peak near the initial 
value of the index $P_{0}=100$.

Fig. 2 Pdf of $V_{T^{*}}$
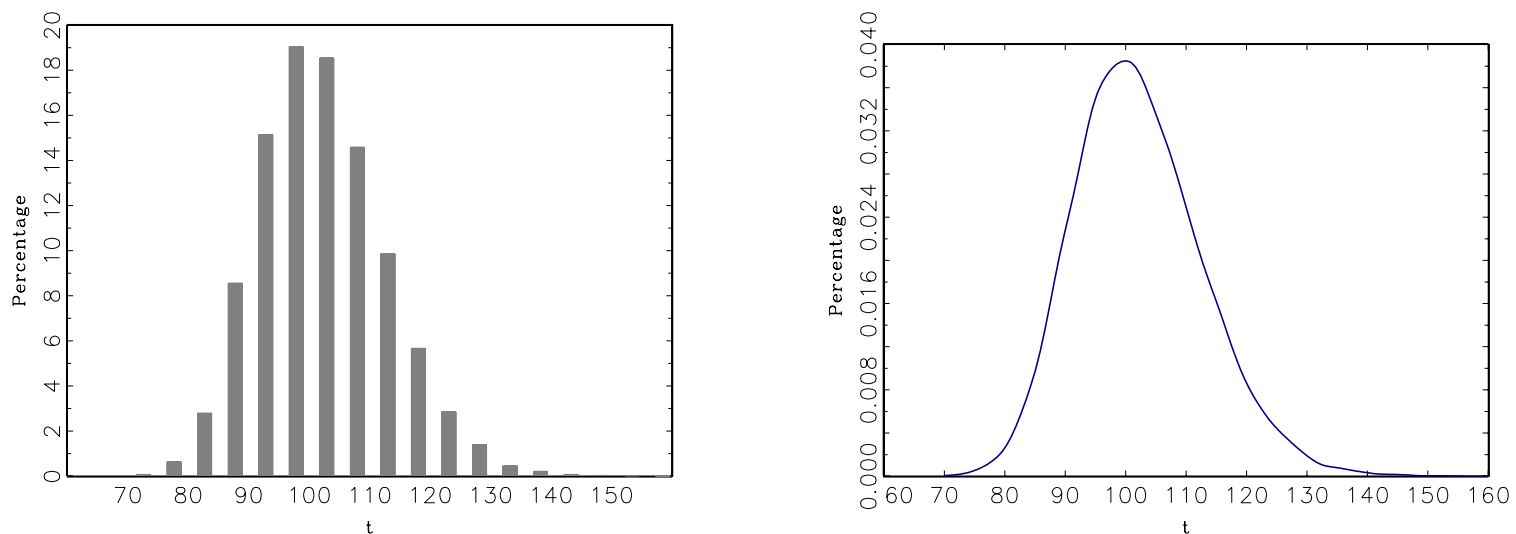

$$
\mu=4.4 \%, \sigma=5 \%, g=3 \%, k=8.4 \%, P_{0}=100, F C F_{0}=100 / 22 .
$$
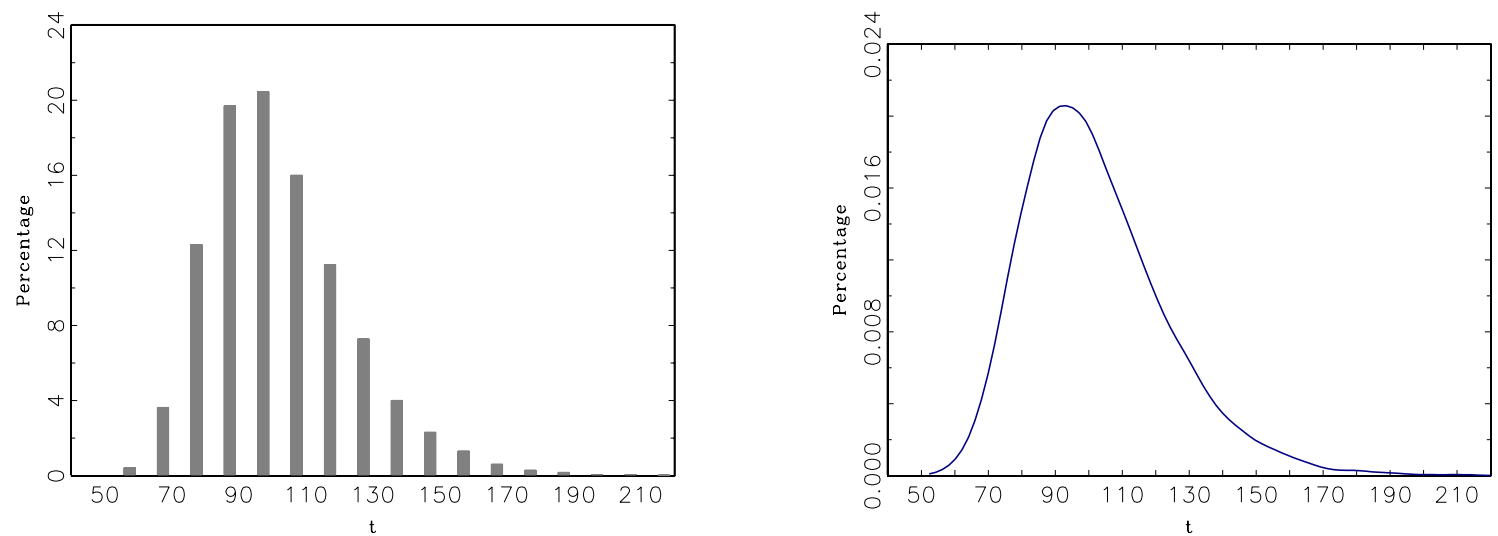

$\mu=4.4 \%, \sigma=10 \%, g=3 \%, k=8.4 \%, P_{0}=100, F C F_{0}=100 / 22$.

\section{Optimal time $T^{* *}$ to sell for a perfectly informed investor}

In this section the investor is assumed to have a perfect foresight about the entire future price path. Paths are random (the investor do not choose the realized path) but, at time 0 , he knows the whole trajectory. Knowing the path, he can optimize on it, which leads to a deterministic solution conditionally to this information. But, taking into account the randomness of the path just before time 0 , the optimal time to sell is a random variable. This corresponds to an 'ideal' case which is not realistic but provides an upward benchmark. First, we examine the distribution of the optimal holding period $T^{* *}$. Second, we indicate the distribution of the optimal value $V_{T^{* *}}$.

\subsection{Probability distribution of $T^{* *}$}

We search for the probability distribution of the random time $T^{* *}$ at which the path of the process $V$ reaches its maximum, where $V$ is the present value of the portfolio. This distribution is not explicitly known but can be simulated. The simulated pdf of 
the real estate portfolio value is illustrated in Figure 3. Two cases are examined:

- $\quad$ The first one corresponds to a relatively small values of the drift $\mu$. and the initial free cash flow $F C F_{0}$.

- $\quad$ The second one corresponds to a higher value of the drift $\mu$ and to a higher value of the initial free cash flow $F C F_{0}$.

Case 1. Early selling:

$$
\mu=0.03, \sigma=0.03, g=0.02, k=0.084, P_{0}=100, F C F_{0}=100 / 22, \bar{T}=20
$$

Case 2. Late selling:

$$
\mu=0.06, \sigma=0.03, g=0.02, k=0.084, P_{0}=100, F C F_{0}=100 / 13, \bar{T}=20
$$

Fig. 3 Simulated pdf of $T^{* *}$ (case 1 and case 2)
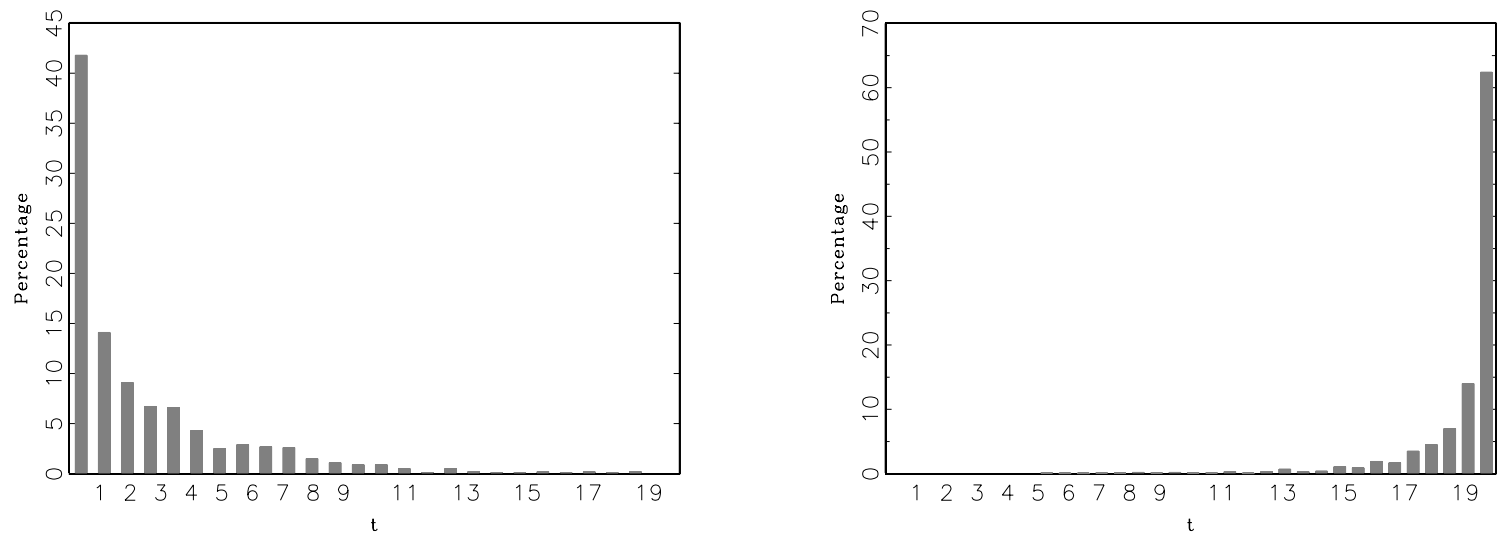

The time $T^{* *}$ at which the discounted portfolio value reaches its maximum depends on the sign of its drift which is an increasing function of both the drift $\mu$. and the initial free cash flow $F C F_{0}$. Hence, if the real estate price return and/or the initial free cash flow $F C F_{0}$ are not high enough, the optimal strategy consists on selling quickly. For both higher discounted cash flows and higher expected discounted index value, the optimal strategy is to sell lately. This shape modification is modelled by the Brownian motion with drift as shown in Appendix A1.

\subsection{Probability distribution of $V_{\mathrm{T} * *}$}

Recall that $T^{* *}$ is random since the investor does not choose the realized path even he knows it (he just observes the trial outcome without selecting it). Now, we search for the probability distribution of the maximum value $V_{T_{* *}}$. We provide an explicit formula by means of a mild approximation. This latter one is justified as shown by Monte Carlo simulations of the true probability distribution (see Figures 4 and 5). Introduce the function $G$ defined by:

$$
G(m, y, t)=1-\frac{1}{2} \operatorname{Erfc}\left(\frac{y}{\sqrt{2 t}}-m \frac{\sqrt{t}}{\sqrt{2}}\right)-\frac{1}{2} e^{2 m y} \operatorname{Erfc}\left(\frac{y}{\sqrt{2 t}}+m \frac{\sqrt{t}}{\sqrt{2}}\right),
$$

where the function $\operatorname{Erfc}$ is given by: 


$$
\operatorname{Erfc}(x)=\frac{2}{\sqrt{\pi}} \int_{x}^{\infty} e^{-u^{2}} d u
$$

Denote also

$$
A(v)=\frac{F C F_{0}}{v}+\mu-k-1 / 2 \sigma^{2}, \text { and } B(v)=\ln \left(\frac{v}{P_{0}}\right) .
$$

Then, the approximated cdf of $V_{T * *}$ is given explicitly by:

$$
P\left[V_{T * *} \leq v\right]=\left\{\begin{array}{c}
0, \text { for } v<P_{0}, \\
G\left(\frac{A(v)}{\sigma}, \frac{B(v)}{\sigma}, \bar{T}\right) \text { for } v>P_{0},
\end{array}\right.
$$

Figure 4 illustrates Relation (15) while Figure 5 shows the quality of the approximation.

Fig. 4 Approximated Pdf of $V_{T^{* *}}$

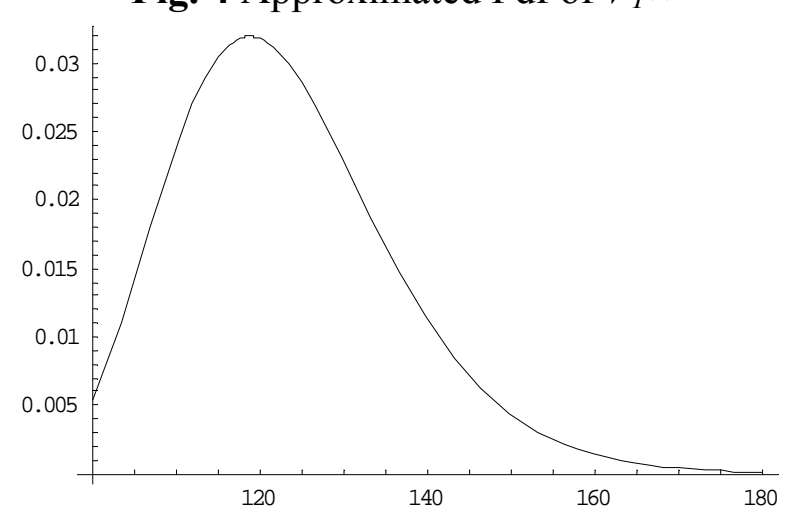

Fig. 5 Simulated Pdf of $V_{T^{* *}}$

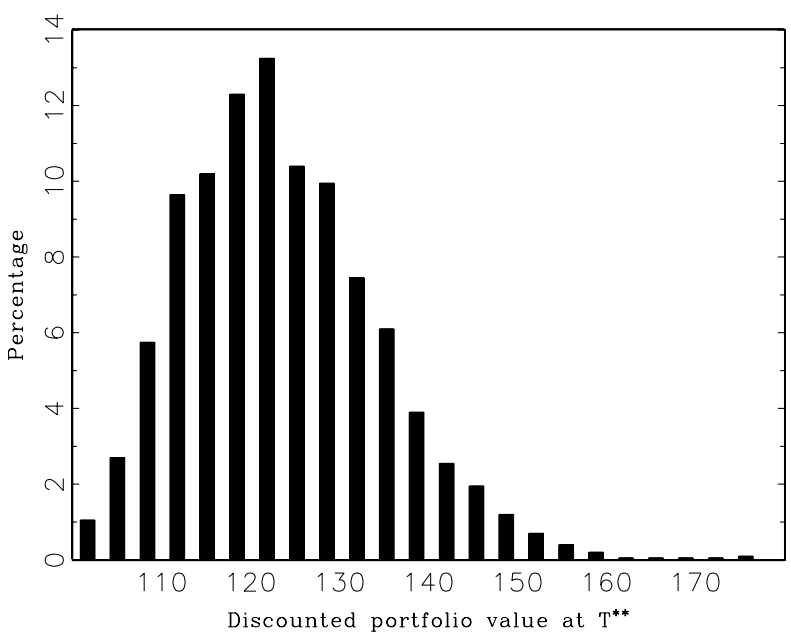

From Figures 4 and 5, we deduce for example that:

- The probability that the real estate portfolio value is higher than $P_{0}$ is equal to 1. Thus, whatever the path, the investor receive at least $P_{0}$. Indeed, if all the future discounted portfolio values are lower than the initial price, he knows he 
has to sell at time 0 and then receives exactly $P_{0}$.

- The median is about $125\left(=+25 \% P_{0}\right)$.

- The probability to receive more than $150\left(=+50 \% P_{0}\right)$ is about $10 \%$.

\subsection{Numerical illustrations}

The distribution of $T^{* *}$ is very sensitive to the volatility parameter, through the terminal value. This is not the case for the solution $T^{*}$. To illustrate this feature, we consider the parameter values:

$$
\mu=0.044, g=0.03, k=0.084, P_{0}=100, F C F_{0}=100 / 22, \bar{T}=20,
$$

and three volatility levels $\sigma=0.0005, \sigma=0.005$ and $\sigma=0.05$.

In Figure 6, each column corresponds to a different volatility level and provides simulated paths of $V_{T^{* *}}$, then the Pdf of $T^{* *}$ and finally the Pdf of $V_{T^{* *}}$.

Fig. 6 Simulated paths of $V$ and Pdf of $T^{* *}$ and $V_{T^{* *}}$

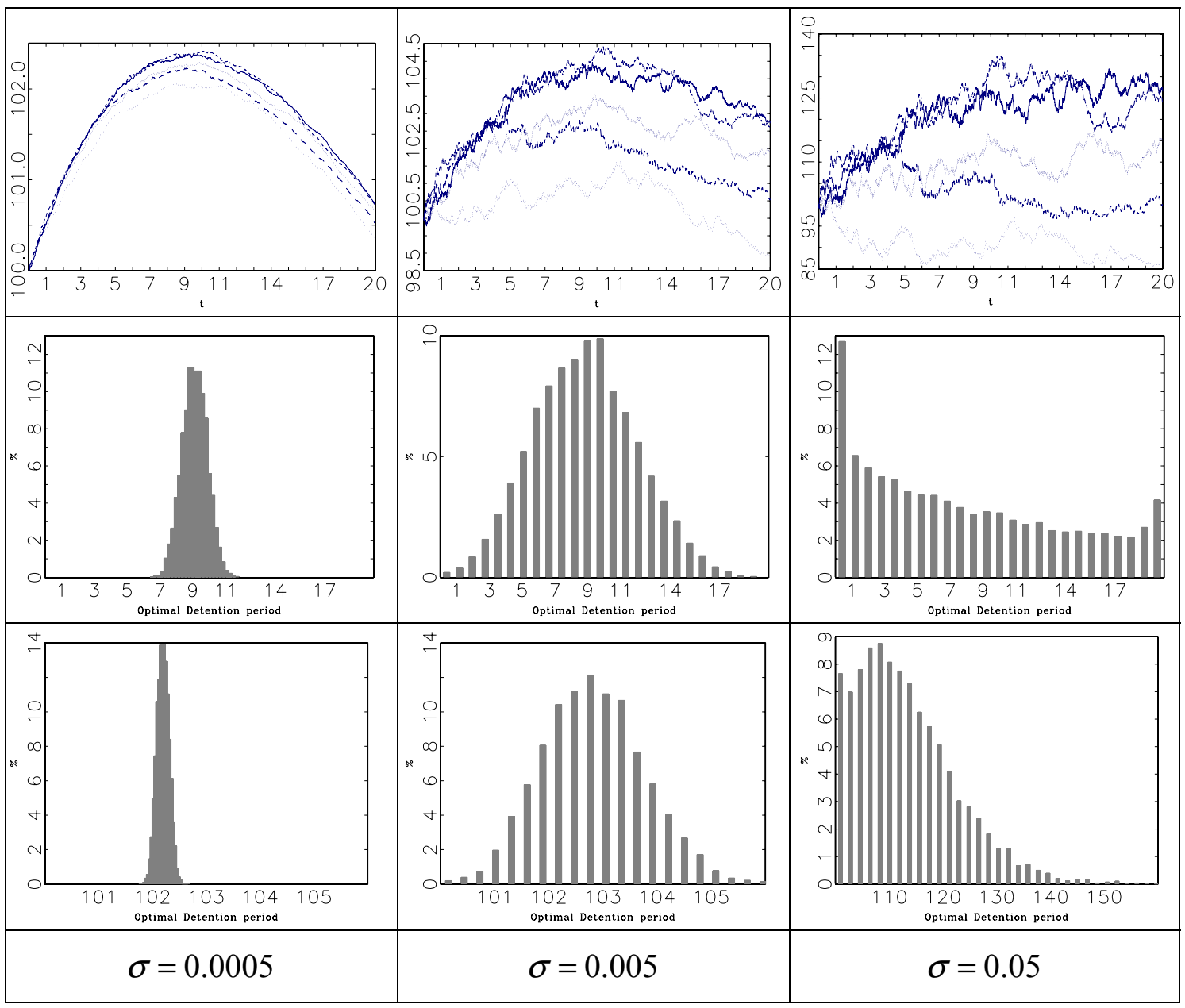

When the volatility is small, the Pdf of $T^{* *}$ is concentrated on the value of $T^{*}$ as expected. When the volatility is increasing, the range of $T^{* *}$ becomes larger. When $\sigma=0.05$, the shape of Pdf is a mix of the Pdf of case 1 and 2 in Figure 3. This is due 
to the Brownian motion (as illustrated in Figure 13 of Appendix A1).

In terms of portfolio value, more volatility implies higher benefits, as the investor in this case is perfectly informed about the path. Note also that when the discounted portfolio values are never higher than the initial price $P_{0}$, the optimal time to sell is $t=0$. Then the optimal value is equal to its smallest value $P_{0}$.

\section{American optimal selling time $\mathrm{T} * * *$}

In this third case, we allow that the investor may choose the optimal time to sell, according to market fluctuations and information from past observations. In this case, he faces an "American" option problem. Recall that the investor preferences are modelled by means of linear utility (standard assumption in real estate literature). At any time $t$ before selling, he compares the present value $P_{t}$ with the maximum of the future value he expects given the available information at time $t$ (mathematically speaking he computes the maximum expected value of his portfolio on all $\mathrm{J}_{t, \bar{T}}-$ measurable stopping times $\tau$ ). It means that he decides to sell at time $t$ only if the portfolio value at this time is higher than the maximal value that he can expect to receive if he does not sell at this time $t$. Thus, he has to compare $P_{t}$ with $\sup _{\tau \in \mathrm{J}_{t, \bar{T}}} E\left[C_{\tau}-C_{t}+P_{\tau} \mid \mathrm{J}_{t}\right]$, where $C_{s}$ denotes the FCF value at time $s$. All the proofs of this section are detailed in Appendix B.

\subsection{The optimal solution $T^{* * *}$}

Intuitively, the optimal time $T^{* * *}$ must be the first time where the asset price $P_{t}$ is "sufficiently" high. At this price level, the future free cash flows (received in case of no sell) will not be high enough to balance an expected index value lower than the price $P_{t}$ at time $t$ (the expected index value decreases with time as the discounted trend $\mu-k$ is negative).

The optimal time $T^{* * *}$ corresponds exactly to the first time at which the asset price $P_{t}$ is higher than the deterministic level $\frac{F C F_{0}}{k-\mu} e^{-(k-g) t}$ (see Appendix B). This result generalizes the case 3 obtained at time $t=0$, in section 2.3, where the investor sells directly the asset if the price $P_{0}$ is higher than $\frac{F C F_{0}}{k-\mu}$. Since the return of the discounted free cash flows is equal to $e^{-(k-g) t}$, the price $P_{t}$ has to be compared with the value $\frac{F C F_{0}}{k-\mu} e^{-(k-g) t}$.

Figure 7 shows the points at which different realized paths of $P_{t}$ cross the deterministic level (black curve). Five paths are simulated for four different volatility levels. 
Fig. 7 Paths of the index value $P_{t}$
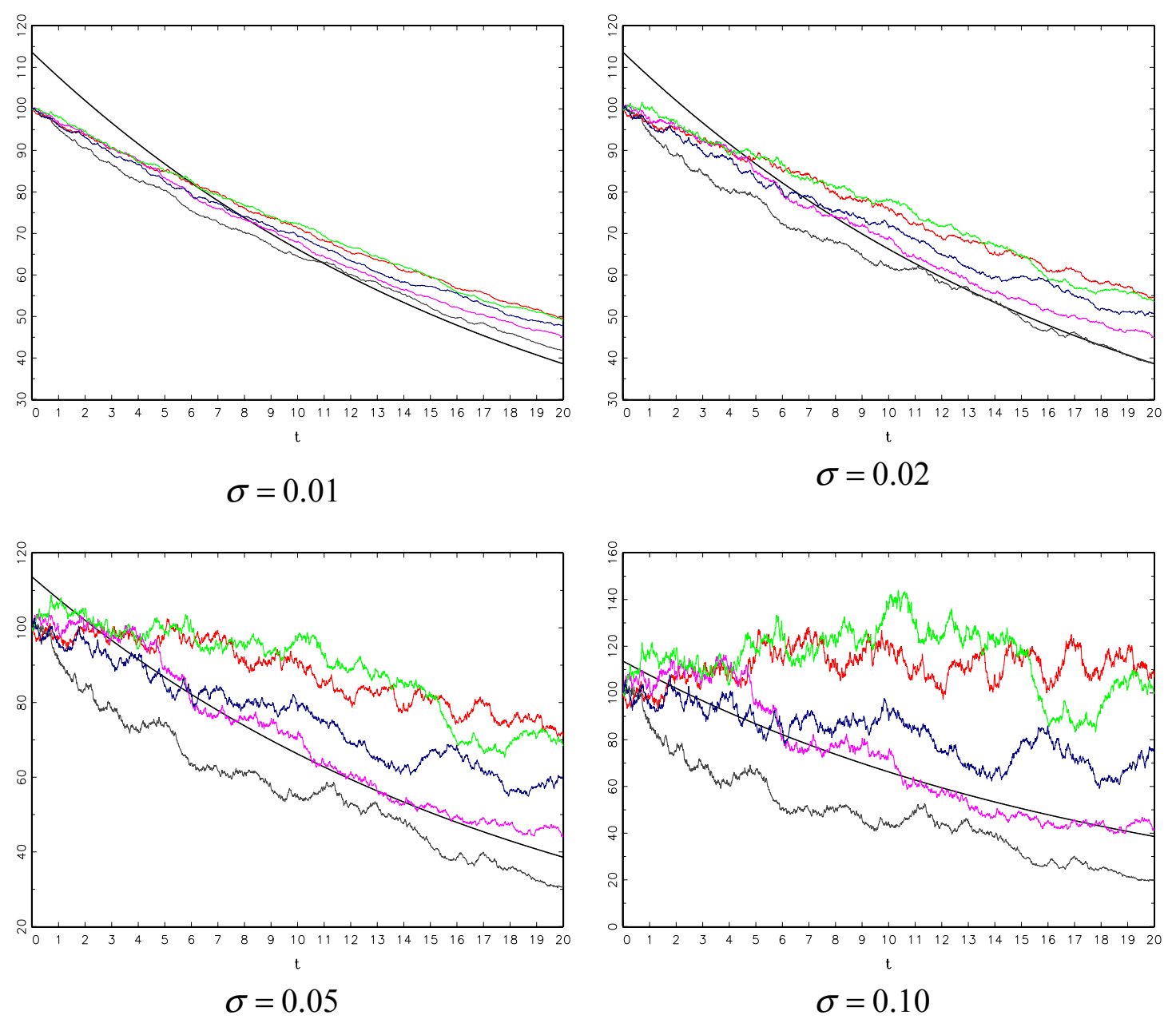

Using the previous characterization of $T^{* * *}$ we can determine its probability distribution. Notations:

$$
m=\frac{\mu-g}{\sigma}-1 / 2 \sigma \text { and } y=\frac{1}{\sigma} \ln \left[\frac{F C F_{0}}{P_{0}(k-\mu)}\right] .
$$

- For the case $\frac{F C F_{0}}{P_{0}(k-\mu)} \leq 1$, we have $T^{* * *}=0$.

- For the case $\frac{F C F_{0}}{P_{0}(k-\mu)}>1$, for any $t<\bar{T}$, we have

$$
\begin{aligned}
& P\left[T^{* * *} \leq t\right]=\frac{1}{2} \operatorname{Erfc}\left(\frac{y}{\sqrt{2 t}}-m \frac{\sqrt{t}}{\sqrt{2}}\right)+\frac{1}{2} e^{2 m y} \operatorname{Erfc}\left(\frac{y}{\sqrt{2 t}}+m \frac{\sqrt{t}}{\sqrt{2}}\right), \\
& P\left[T^{* * *}=\bar{T}\right]=1-\frac{1}{2} \operatorname{Erfc}\left(\frac{y}{\sqrt{2 \bar{T}}}-m \frac{\sqrt{\bar{T}}}{\sqrt{2}}\right)-\frac{1}{2} e^{2 m y} \operatorname{Erfc}\left(\frac{y}{\sqrt{2 \bar{T}}}+m \frac{\sqrt{\bar{T}}}{\sqrt{2}}\right) .
\end{aligned}
$$

As illustrated in Figure 8 (for $\sigma=10 \%, \bar{T}=20$ ), the probability that the optimal American time to sell $T^{* * *}$ may be not negligible (about $15 \%$ for this numerical case, which corresponds to the size of the jump of the cdf). 
Fig. 8 Cdf of $T^{* * *}$

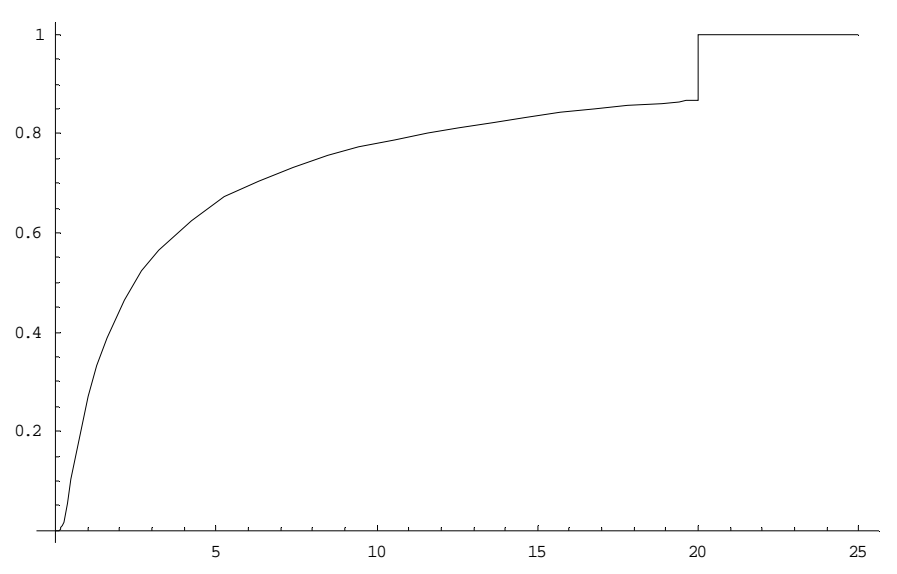

\subsection{The portfolio value $V_{\mathrm{T}^{+4+4}}$ and its $c d f$}

The portfolio value $V_{T^{* * * *}}$ can be determined by using the fact that, if the optimal time to sell $T^{* * *}$ is before the maturity $\bar{T}$, then the index value $P_{T^{* * *}}$ is equal to the deterministic level $\frac{F C F_{0}}{k-\mu} e^{-(k-g) T^{* * *}}$. Consequently, we get:

$$
\begin{aligned}
& V_{T^{* * *}}=\frac{F C F_{0}}{(k-g)}\left(1+e^{-(k-g) T^{* * *}} \frac{(\mu-g)}{(k-\mu)}\right), \text { if } T^{* * * *}<\bar{T} \\
& V_{T^{* * * *}}=\frac{F C F_{0}}{(k-g)}\left(1-e^{-(k-g) \bar{T}}\right)+P_{0} \exp \left[\left(\mu-k-1 / 2 \sigma^{2}\right) \bar{T}+\sigma W_{\bar{T}}\right], \text { if } T^{* * *}=\bar{T}
\end{aligned}
$$

The previous determination of the value $V_{T^{* * *}}$ allows the explicit (and exact) computation of its cdf.

Denoting:

$$
z(v)=\frac{1}{\sigma} \ln \left[\frac{v-\frac{F C F_{0}}{(k-g)}\left(e^{(k-g) \bar{T}}-1\right)}{P_{0}}\right] ; g(v)=\frac{1}{k-g} \ln \left[\frac{\mu-g}{k-\mu} \times \frac{\frac{F C F_{0}}{k-g}}{v-\frac{F C F_{0}}{k-g}}\right],
$$

the cdf of $V_{T^{* * *}}$ is defined by:

- If $v \leq \frac{F C F_{0}}{k-g}\left(1-e^{-(k-g) \bar{T}}\right)$

$$
F_{V_{T^{* * *}}}(v)=0
$$

- If $\frac{F C F_{0}}{k-g}\left(1-e^{-(k-g) \bar{T}}\right)<v<\left(\frac{F C F_{0}}{k-g}\right)\left(1+\frac{\mu-g}{k-\mu} e^{-(k-g) \bar{T}}\right)$,

$$
F_{V_{T^{* *+}}}(v)=N\left(\frac{z(v)-m \bar{T}}{\sqrt{\bar{T}}}\right)-e^{2 y m} N\left(\frac{z(v)-(m \bar{T}+2 y)}{\sqrt{\bar{T}}}\right)
$$




$$
\begin{aligned}
& \text { - If }\left(\frac{F C F_{0}}{k-g}\right)\left(1+\frac{\mu-g}{k-\mu} e^{-(k-g) \bar{T}}\right) \leq v \leq \frac{F C F_{0}}{k-g}\left(1+\frac{\mu-g}{k-\mu}\right) \text {, } \\
& F_{V_{T^{* * * *}}}(v)=G(m, y, g(v))-G(m, y, \bar{T}) \\
& +N\left(\frac{z(v)-m \bar{T}}{\sqrt{\bar{T}}}\right)-e^{2 y m} N\left(\frac{-y-m \bar{T}}{\sqrt{\bar{T}}}\right)-\left[N\left(\frac{z(v)-m \bar{T}}{\sqrt{2 \bar{T}}}\right)-N\left(\frac{y-m \bar{T}}{\sqrt{2 \bar{T}}}\right)\right] \\
& \text { - If } \frac{F C F_{0}}{k-g}\left(1+\frac{\mu-g}{k-\mu}\right)<v \\
& \begin{array}{c}
F_{V_{T^{*+*}}}(v)=1-G(m, y, \bar{T}) \\
+N\left(\frac{z(v)-m \bar{T}}{\sqrt{\bar{T}}}\right)-e^{2 y m} N\left(\frac{-y-m \bar{T}}{\sqrt{\bar{T}}}\right)-\left[N\left(\frac{z(v)-m \bar{T}}{\sqrt{2 \bar{T}}}\right)-N\left(\frac{y-m \bar{T}}{\sqrt{2 \bar{T}}}\right)\right] .
\end{array}
\end{aligned}
$$

\section{Comparison of the three optimal strategies and the buy-and hold one}

We examine both the probability distributions of the optimal times to sell $T^{*}, T^{* *}$ and $T^{* * * *}$ and the corresponding discounted portfolio values $V_{T^{*}}, V_{T^{* *}}$ and $V_{T^{* * * *}}$. We introduce also the comparison with the buy-and hold portfolio $V_{\bar{T}}$. The sensitivity analysis is done with respect to the volatility $\sigma$ and to the maturity $\bar{T}$. Note that, since the portfolio value $V_{T^{* *}}$ dominates the other ones, its cdf is always below the cdf of the others ("first order stochastic dominance").

The previous parameter values, $\mu=0.044, g=0.03, k=0.084, P_{0}=100, \quad$ and $F C F_{0}=100 / 22$, are considered.

\subsection{Cdf of $T^{* *}$ and $T^{* * *}$, according to the volatility $\sigma$}

The maturity $\bar{T}$ is equal to 20 and then we have $T^{*}=9.13$. Graphical results are presented in Figure 9.

- For small volatility level, the cdf of $T^{* * *}$ is rather concentrated near $T^{*}$, whereas, for higher values, the shape of the cdf of $T^{* * *}$ looks similar to the cdf of $T^{* *}$.

- Additionally, for the American case, the probability that the selling occurs at maturity $\left(P\left[T^{* * *}=\bar{T}\right]\right)$ is almost equal to 0 , for small volatility levels. However, for higher volatility values, this probability is about $15 \%$. Indeed, this probability converges to a limit which is equal to $1-e^{-\ln \left[\frac{F C F_{0}}{P_{0}(k-\mu)}\right]}$.

- Moreover, higher the volatility, higher the probability of smaller American optimal times, as usually observed empirically (see Collett et al., 2003). 
Fig. $9 \mathrm{Cdf}$ of $T^{* *}$ and $T^{* * *}$ with respect to $\sigma$
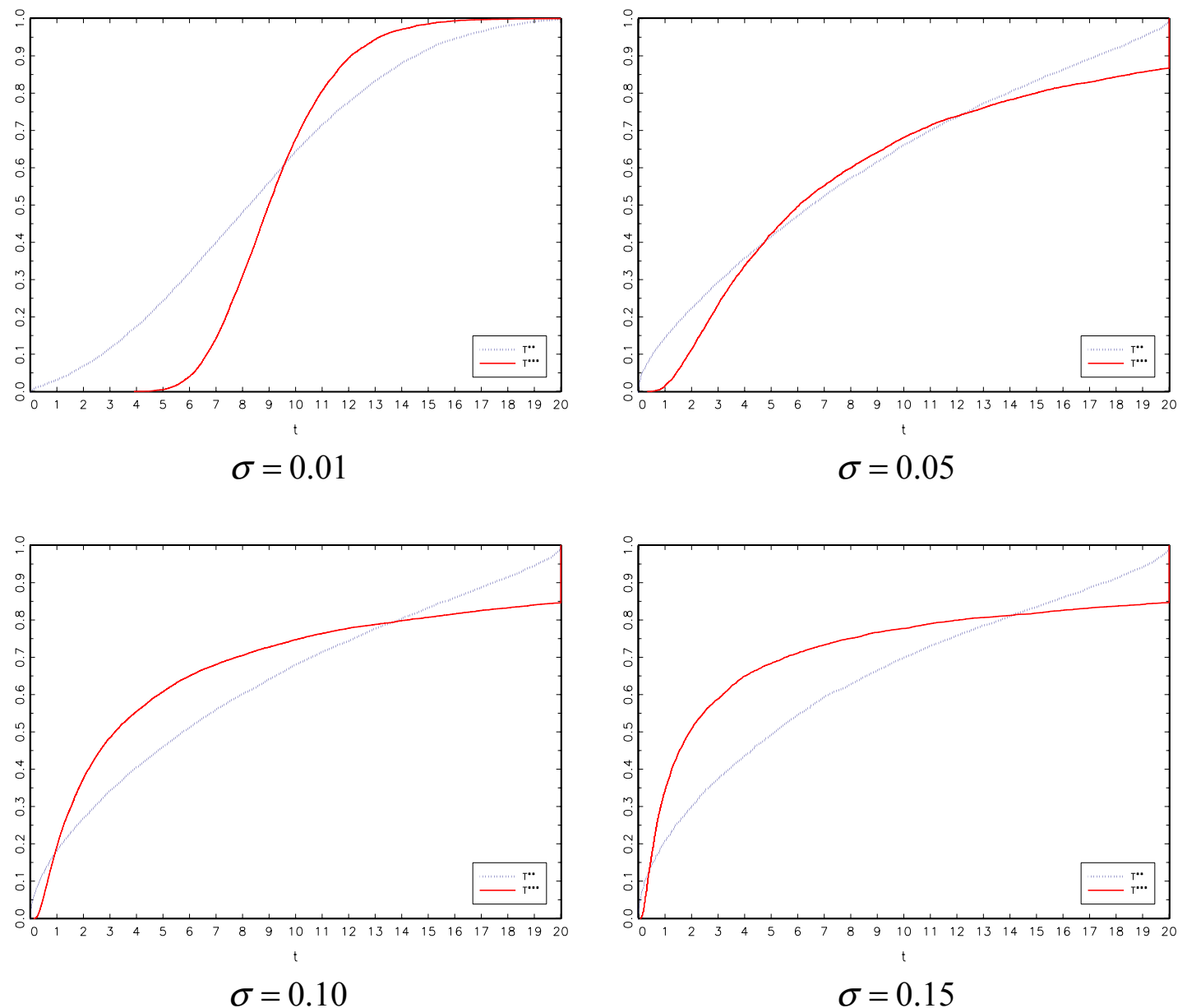

5.2. Cdf of $V_{\bar{T}}, V_{T^{*}} V_{T^{* *}}$ and $V_{T^{* * *}}$ with respect to $\sigma$

We assume also that $\bar{T}=20$ and then $T^{*}=9.13$. Graphical results are presented in Figure 10.

- For small volatility level, the cdf of $V_{T^{* * *}}$ is almost equal to the cdf of $V_{T^{*}}$. For higher values, two components of the cdf of $V_{T^{* * * *}}$ can be clearly identified. The smallest values of $V_{T^{* * *+}}$ (approximately values up to 95$)$, correspond to an optimal time to sell $T^{* * *}$ equal to the maturity $(\bar{T}=20)$ : the discounted portfolio values were too small so that it was not optimal to sell before maturity.

- For any given threshold $L$ smaller than 105, the probability that the American portfolio value $V_{T^{* * *}}$ is smaller than $L$, is always weaker than the corresponding value for $V_{T^{*}}$. The American option approach leads to smaller losses, when comparing the discounting portfolio value to the initial price $\left(P_{0}=100\right)$. More generally, this approach prevents from large fluctuations of the price dynamics. When the index 
price falls, the American criterion provides protection against downside risk. When the index price rises significantly, this approach leads to an earlier sell, in order to insure the profit of this increase. If after the sell the index dynamics goes down, the investor did right avoiding then lower profits or even losses. Finally, if after the sell the index dynamics goes up, his profit would have been higher if he had not sold.

- The shape and the values of the cdf of $V_{\bar{T}}$ (the buy-and hold approach), look like the cdf of $V_{T^{*}}$. except for very small volatilities.

Fig. $10 \mathrm{Cdf}$ of $V_{\bar{T}}, V_{T^{*}} V_{T^{* *}}$ and $V_{T^{* * *}}$ with respect to $\sigma$
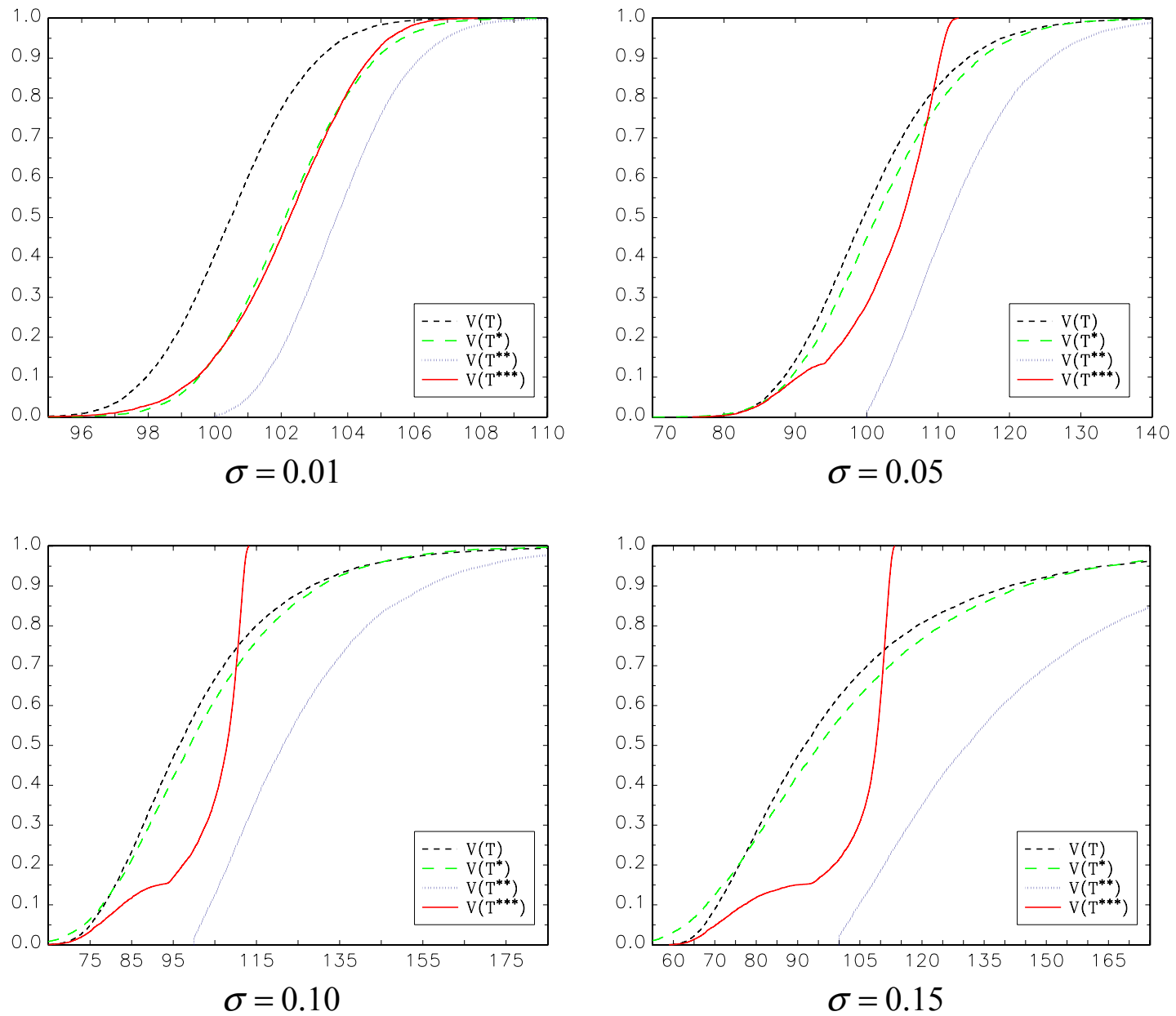

\subsection{Cdf of $T^{* *}$ and $T^{* * * *}$ with respect to $\bar{T}$}

Graphical results are presented in Figure $11(\sigma=5 \%)$.

- In the American option approach, the probability to sell at maturity (given by $P\left[T^{* * *}=\bar{T}\right]$ ) is almost equal to $90 \%$ for small maturity (see the jump of the cdf for $\bar{T}=2$ ), whereas for long maturity, it becomes very smaller (around $6 \%$, for $\bar{T}=30$ ). 
- For the perfectly informed case, the probability to exercise at maturity is null $\left(P\left[T^{* *}=\bar{T}\right]=0\right)$, whatever the maturity. The shape of the cdf of $T^{* * *}$ looks similar to the cdf of $T^{* *}$ for high maturity.

Fig. $11 \mathrm{Cdf}$ of $T^{* *}$ and $T^{* * *}$ with respect to $\bar{T}$
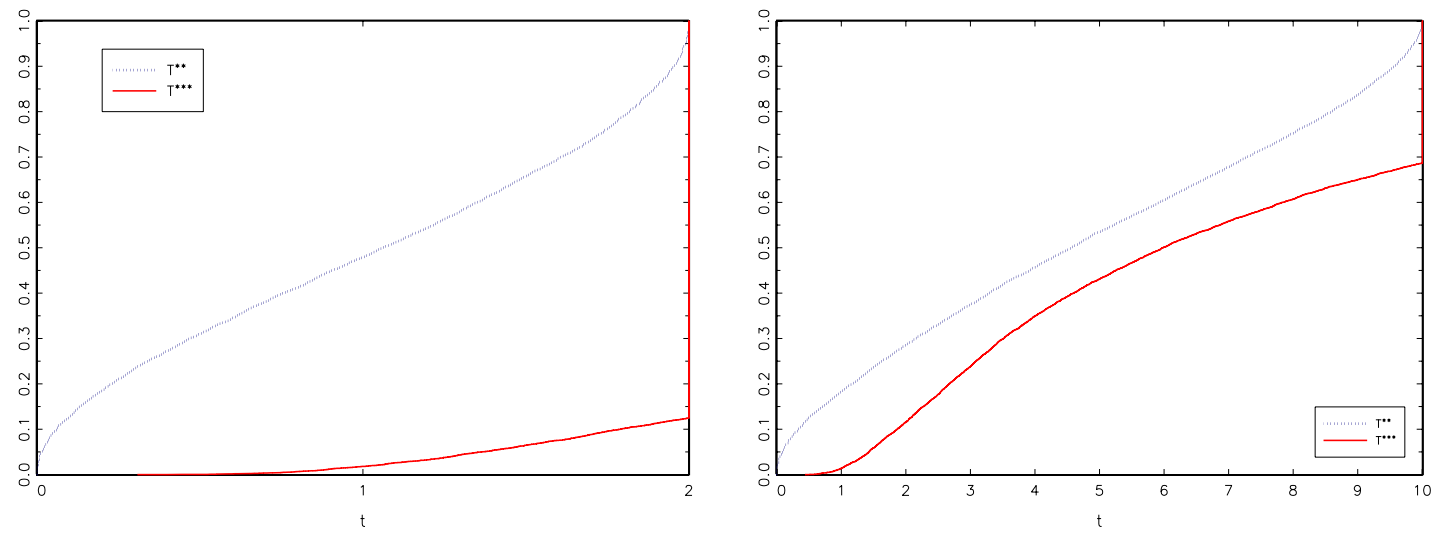

$$
\bar{T}=2
$$

$$
\bar{T}=10
$$
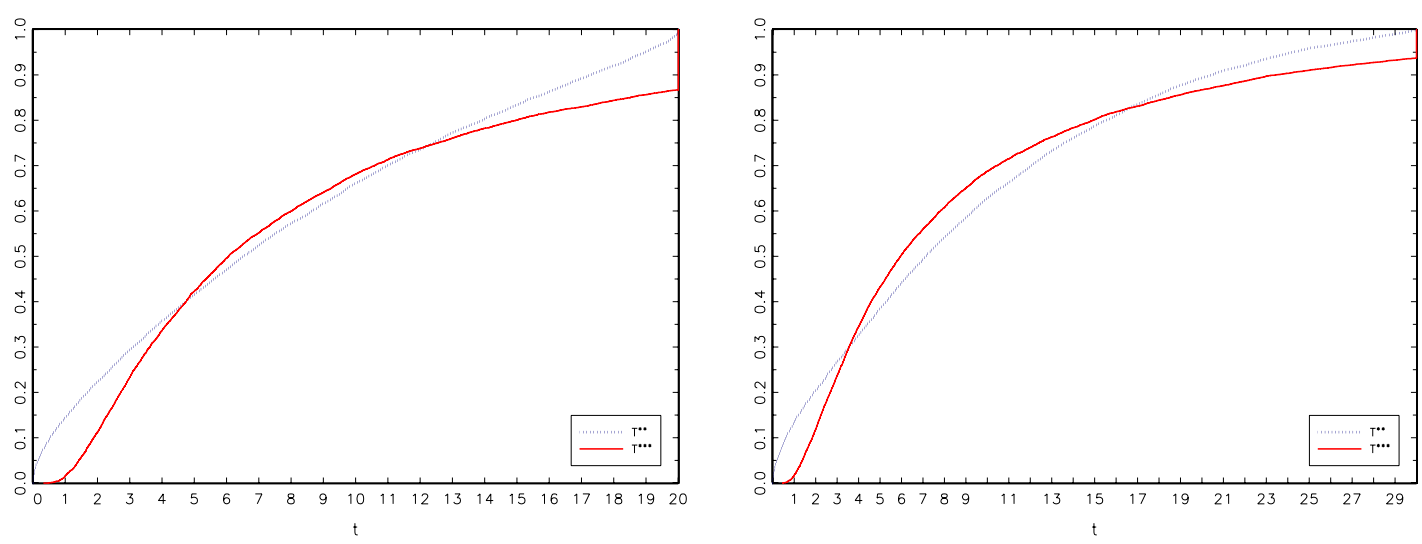

$$
\bar{T}=20
$$

$$
\bar{T}=30
$$

\subsection{Cdf of $V_{\bar{T}}, V_{T^{*}} V_{T^{* *}}$ and $V_{T^{* * *}}$ according to the maturity $\bar{T}$}

Graphical results are presented in Figure $12(\sigma=5 \%)$.

- For small maturity, the cdf of $V_{T^{* * *}}$ is almost equal to the cdf of $V_{T^{*}}$. The difference comes from the probability to sell before maturity, which modifies the probability to receive high portfolio values.

- For longer maturities, this difference is more important. The cumulative distribution functions diverge more and more, except for the smallest portfolio values.

- Whatever the maturity, the numerical upper bound of $V_{T^{* * *}}$ is around 115 (this bound is still present for other volatility values, as seen in Figure 10). Hence, higher portfolio values may be obtained with the $T^{*}$ case. When the a priori optimal time to sell $T^{*}$ is higher than the maturity $\left(T^{*}>\bar{T}\right)$, the portfolio values $V_{\bar{T}}$ corresponding to the buy- 
and-hold strategy are exactly the same as the values $V_{T^{*}}$ of the strategy $T^{*}$. When $T^{*}<\bar{T}$, higher the maturity, higher the difference between the cdfs of $V_{\bar{T}}$ and $V_{T^{*}}$.

Fig. 12 Cdf of $V_{\bar{T}}, V_{T^{*}}, V_{T^{* *}}$ and $V_{T^{* * *}}$ with respect to $\bar{T}$
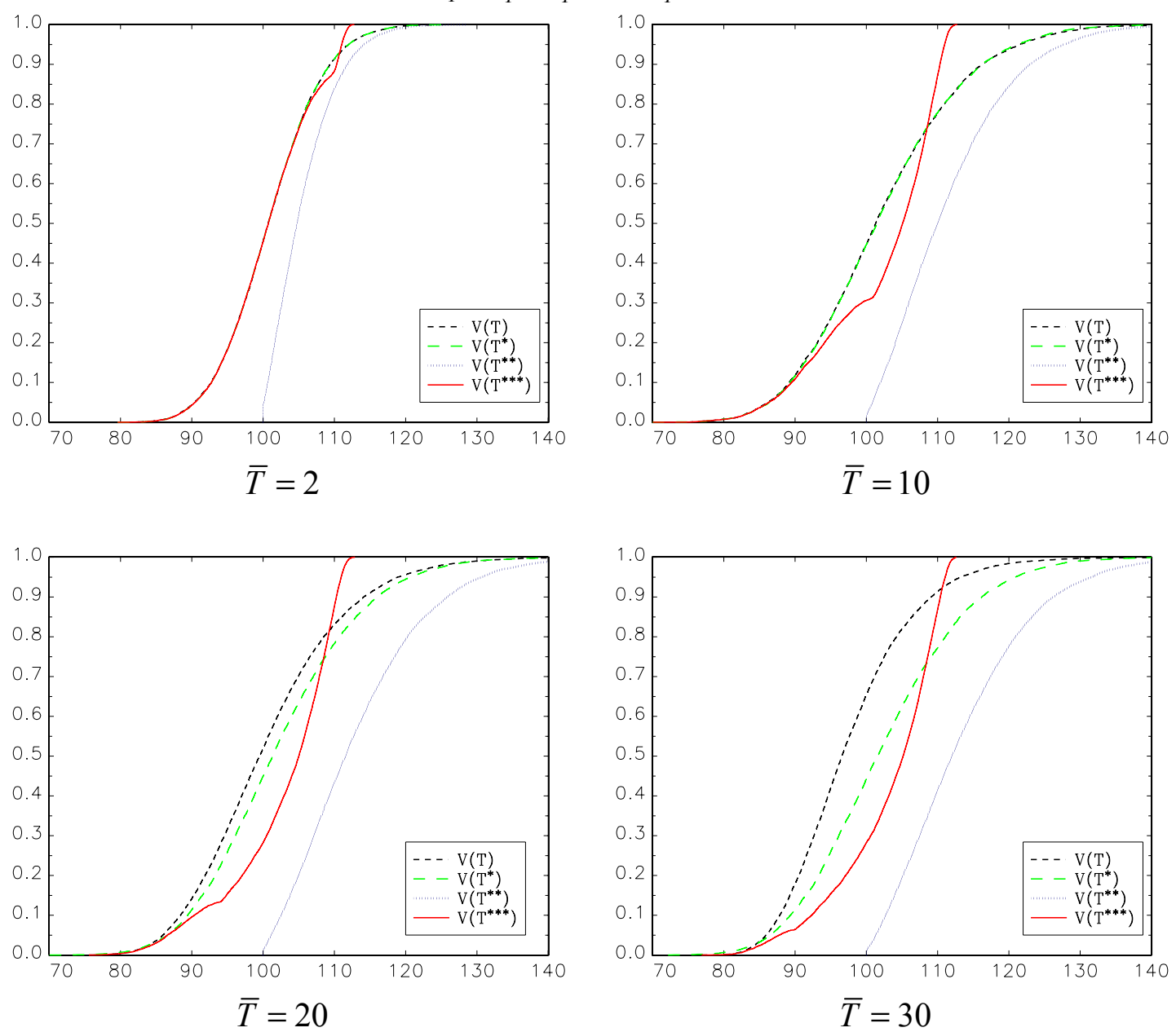

\section{Conclusion}

This paper proves that real estate portfolio value strongly depends on the optimal time to sell. Three kinds of such optimal times are considered. The first one implicitly assumes that the investor can only choose the optimal time to sell at the initial date. However, generally such a solution is not time consistent: the same computation of optimal time to sell at a future date leads to a different solution. A second one corresponds to a perfectly informed investor. This is an upward benchmark but not too realistic. Finally, a more "rational" approach is introduced to take account of intertemporal management and cumulative information. This is the American option framework. For each of these models, the optimal times to sell and portfolio values are explicitly determined (an approximation is used for the "perfectly informed" case) and also simulated. We compare the solutions, using various parameter values of the real estate markets, in particular the volatility index and the portfolio maturity. The American approach allows the reduction of the probability to get small portfolio values. This property is reinforced both when the volatility and the maturity are increasing. 


\section{Appendix}

\section{Appendix A: The case T**}

\section{A.1. Comments about the pdf $f_{T^{* *}}$.}

This pdf is not known since the process $V$ to be maximized on the time interval $[0, \bar{T}]$ has the following form:

$$
V_{t}=c\left(1-e^{-a t}\right)+P_{0} e^{b t+\sigma W_{t}},
$$

where $a$ and $b$ are non negative constant.

However, for a 55process $X$ defined as a geometric Brownian motion with drift by

$$
X_{t}=X_{0} e^{b t+\sigma W_{t}} \text { with } X_{0}>0 \text { and } \sigma>0 \text {, }
$$

the pdf of $T^{* *}$ is known. Indeed, note that $X_{t}=X_{0}\left(e^{\frac{b}{\sigma} t+W_{t}}\right)^{\sigma}$. Therefore, the maximization of $X$ is equivalent to the maximization of the process $Y$ defined by $Y_{t}=e^{\frac{b}{\sigma} t+W_{t}}$.

Recall the probability density function $f_{T_{1}}$ of the first time $T_{1}$ before $\bar{T}$ at which a Brownian motion with drift $W^{(m)}$ reaches its maximum (see Borodin and Salminen, 2002). Let $W^{(m)}$ be defined by:

$$
W_{t}^{(m)}=m t+W_{t},
$$

where $W$ is a standard Brownian motion. Then, the probability density of the random time $T_{1}$ at which the path of the process $W^{(m)}$ reaches its maximum is given by:

$$
\begin{gathered}
f_{T_{1}}(v)= \\
\left(\frac{e^{-m^{2} v / 2}}{\sqrt{\pi v}}+\frac{m}{\sqrt{2}} \operatorname{Erfc}\left[-\frac{m \sqrt{2}}{\sqrt{2}}\right]\right)\left(\frac{e^{-m^{2}(\bar{T}-v) / 2}}{\sqrt{\pi(\bar{T}-v)}}-\frac{m}{\sqrt{2}} \operatorname{Erfc}\left[\frac{m \sqrt{(\bar{T}-v)}}{\sqrt{2}}\right]\right) .
\end{gathered}
$$

Figure 13 illustrates the inversion of the cdf curves, as observed in Figure 3 for the simulated cdf of $T^{* *}$. We consider two cases:

1) $m<0(m=-1, T=30$ years $)$

Due to the negative drift, the maximum is early achieved.

2) $m>0(m=+1, T=30$ years $)$

Due to the positive drift, the maximum is tardily achieved. 

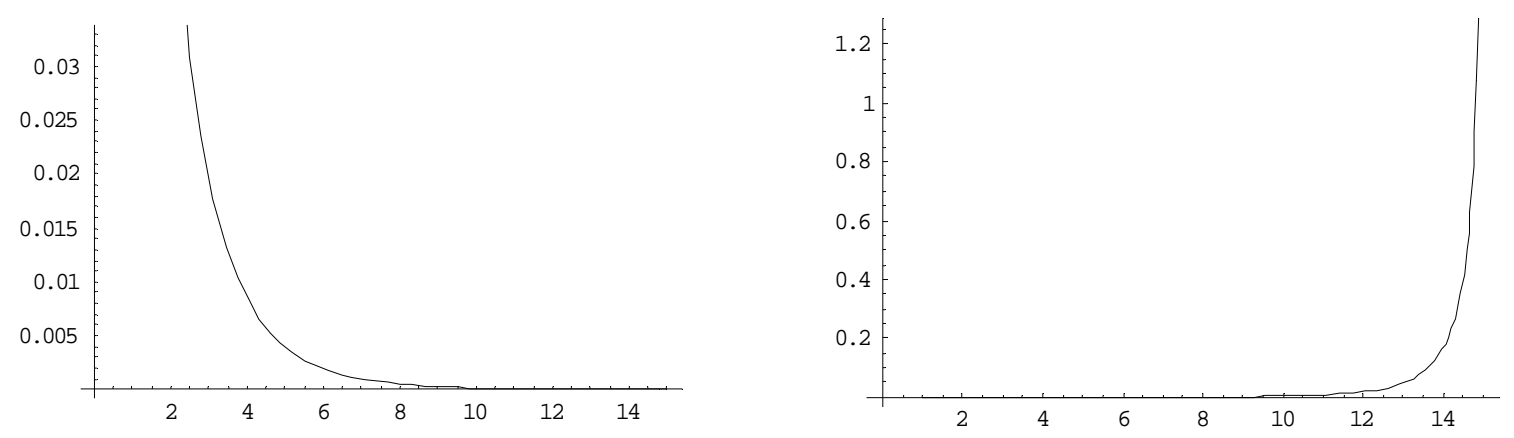

Fig. 13 Pdf approximation of $T^{* *}$ (negative and positive drift)

A.2. Computation of the cdf $f_{V_{T^{* * *}}}$.

The approximated cdf of $V_{T * *}$ is given by:

$$
\begin{gathered}
P\left[\operatorname{Sup}_{0 \leq t \leq \bar{T}} V_{t} \leq v\right]= \\
P\left[\forall t \leq \bar{T}, \exp \left[\left(\mu-k-1 / 2 \sigma^{2}\right) t+\sigma W_{t}\right] \leq \frac{(v-c) e^{a t}+c}{P_{0}}\right],
\end{gathered}
$$

where $a=k-g$ and $c=F C F_{0} /(k-g)$.

Denote:

Then, the inequality

$$
\alpha=\ln \left[\frac{P_{0}}{c}\right] \text {, and } \beta=\ln \left[\frac{v}{c}-1\right]
$$

$$
\exp \left[\left(\mu-k-1 / 2 \sigma^{2}\right) t+\sigma W_{t}\right] \leq \frac{(v-c) e^{a t}+c}{P_{0}}
$$

is equivalent to:

$$
\exp \left[\alpha+\left(a+\mu-k-1 / 2 \sigma^{2}\right) t+\sigma W_{t}\right] \leq e^{\beta+a t}+1 .
$$

Consider the following approximation:

$$
1+e^{\beta+x}=e^{y} \text { with } y \simeq \ln \left(1+e^{\beta}\right)+\left(\frac{e^{\beta}}{1+e^{\beta}}\right) x .
$$

Then:

$$
\begin{gathered}
P\left[\operatorname{Sup}_{0 \leq t \leq \bar{T}} V_{t} \leq v\right] \simeq \\
P\left[\forall t \leq \bar{T}, \exp \left[\alpha+\left(a+\mu-k-1 / 2 \sigma^{2}\right) t+\sigma W_{t}\right] \leq \exp \left[\ln \left(1+e^{\beta}\right)+\left(\frac{e^{\beta}}{1+e^{\beta}}\right) a t\right]\right] .
\end{gathered}
$$

Denote:

$$
A(v)=a+\mu-k-1 / 2 \sigma^{2}-\left(\frac{e^{\beta}}{1+e^{\beta}}\right) a \text { and } B(v)=\ln \left(1+e^{\beta}\right)-\alpha=\ln \left(\frac{v}{P_{0}}\right) .
$$

Note that we have:

$$
A(v)=\frac{a c}{v}+\mu-k-1 / 2 \sigma^{2} \text { and } B(v)=\ln \left(\frac{v}{P_{0}}\right) .
$$

Therefore:

$$
P\left[\operatorname{Sup}_{0 \leq t \leq \bar{T}} V_{t} \leq b\right]=P\left[\forall t \leq \bar{T}, \frac{A(v)}{\sigma} t+W_{t} \leq \frac{B(v)}{\sigma}\right] .
$$


Recall (see Borodin and Salminen, 2002, p. 250) that the maximum value of the Brownian motion with drift $W^{(m)}$ before $\bar{T}$ has the following cdf:

$$
\begin{gathered}
P\left[\operatorname{Sup}_{0 \leq t \leq \bar{T}} W_{t}^{(m)} \leq y\right]= \\
G(m, y, \bar{T})=1-\frac{1}{2} \operatorname{Erfc}\left(\frac{y}{\sqrt{2 \bar{T}}}-m \frac{\sqrt{\bar{T}}}{\sqrt{2}}\right)-\frac{1}{2} e^{2 m y} \operatorname{Erfc}\left(\frac{y}{\sqrt{2 \bar{T}}}+m \frac{\sqrt{\bar{T}}}{\sqrt{2}}\right) .
\end{gathered}
$$

Consequently, we deduce:

Denote:

$$
\left\{\begin{array}{c}
\text { If } v>P_{0}, P\left[\operatorname{Sup}_{0 \leq t \leq \bar{T}} V_{t} \leq v\right]=G\left(\frac{A(v)}{\sigma}, \frac{B(v)}{\sigma}, \bar{T}\right), \\
\text { If } v<P_{0}, P\left[\operatorname{Sup}_{0 \leq t \leq \bar{T}} V_{t} \leq v\right]=0 .
\end{array}\right.
$$

Consider the following approximation:

$$
\alpha=\ln \left[\frac{P_{0}}{c}\right], \text { and } \gamma=\ln \left[1-\frac{v}{c}\right] .
$$

$$
1-e^{\gamma+x}=e^{y} \text { with } y \simeq \ln \left(1-e^{\gamma}\right)+\left(-\frac{e^{\gamma}}{1-e^{\gamma}}\right) x .
$$

Then the inequality

$$
\exp \left[\left(\mu-k-1 / 2 \sigma^{2}\right) t+\sigma W_{t}\right] \leq \frac{(v-c) e^{a T}+c}{P_{0}}
$$

is equivalent to:

$$
\exp \left[\alpha+\left(a+\mu-k-1 / 2 \sigma^{2}\right) t+\sigma W_{t}\right] \leq 1-e^{\gamma+a T}
$$

Thus, we deduce the same equality as previously:

$$
P\left[\operatorname{Sup}_{0 \leq t \leq \bar{T}} V_{t} \leq v\right]=P\left[\forall t \leq \bar{T}, \frac{A(v)}{\sigma} t+W_{t} \leq \frac{B(v)}{\sigma}\right] .
$$

Consequently, we have:

$$
\left\{\begin{array}{c}
\text { If } v>P_{0}, P\left[\operatorname{Sup}_{0 \leq t \leq \bar{T}} V_{t} \leq v\right]=G\left(\frac{A(v)}{\sigma}, \frac{B(v)}{\sigma}, \bar{T}\right), \\
\text { If } v<P_{0}, P\left[\operatorname{Sup}_{0 \leq t \leq \bar{T}} V_{t} \leq v\right]=0 .
\end{array}\right.
$$

Finally, the cdf of the maximum value $V_{T * *}$ is (approximately) given by:

$$
P\left[V_{T * *} \leq v\right]=\left\{\begin{array}{c}
0, \text { for } v<P_{0}, \\
G\left(\frac{A(v)}{\sigma}, \frac{B v)}{\sigma}, \bar{T}\right) \text { for } v>P_{0},
\end{array}\right.
$$

Using the definitions of coefficients $A, B$, and $c$, the cdf of $V_{T * *}$ is deduced. 


\section{Appendix B: The American case $T^{* * *}$}

\section{B.1. The American option problem}

Denote by $\mathrm{V}(x, t)$ the following value function:

$$
\mathrm{V}(x, t)=\sup _{\tau \in F_{t, \bar{T}}} E\left[C_{\tau}-C_{t}+P_{\tau} \mid P_{t}=x\right] .
$$

Note that we always have $\mathrm{V}(x, t) \geq x$, since $\tau=t \in \mathrm{J}_{t, \bar{T}}$ and, in that case, $\mathrm{V}(x, t)=x$. As usual for American options ${ }^{6}$, two "regions" have to be considered:

- The continuity region:

$$
C=\left\{(x, t) \in R^{+} \times[0, \bar{T}] \mid \mathrm{V}\left(P_{t}, t\right)>P_{t}\right\}
$$

- $\quad$ The stopping region:

$$
S=\left\{(x, t) \in R^{+} \times[0, \bar{T}] \mid \mathrm{V}\left(P_{t}, t\right)=P_{t}\right\}
$$

The first optimal stopping time $T_{t}^{* * *}$ after time $t$ is given by

$$
T_{t}^{* * *}=\inf \left\{u \in[t, \bar{T}] \mid \mathrm{V}\left(P_{u}, u\right)=P_{u}\right\} .
$$

Then:

$$
T_{t}^{* * *}=\inf \left\{u \in[t, \bar{T}] \mid P_{t} \notin C\right\} .
$$

\section{B.2. Computation of the value function $\mathrm{V}$}

To determine $T_{t}^{* * *}$, we have to calculate $\mathrm{V}(x, t)$.

Note that $\left(\exp \left[\left(-1 / 2 \sigma^{2}\right) t+\sigma W_{t}\right]\right)$ is a martingale with respect to the filtration $\mathrm{J}_{t}$. Therefore:

$$
E\left[\exp \left[\left(-1 / 2 \sigma^{2}\right) \tau+\sigma W_{\tau}\right] \mid \mathrm{J}{ }_{t}\right]=\exp \left[\left(-1 / 2 \sigma^{2}\right) t+\sigma W_{t}\right]
$$

Moreover, since

$$
P_{t}=P_{0} \exp \left[\left(\mu-k-1 / 2 \sigma^{2}\right) t+\sigma W_{t}\right]
$$

we have:

$$
\begin{gathered}
E\left[C_{\tau}-C_{t}+P_{\tau} \mid P_{t}=x\right]= \\
E\left[C_{\tau}-C_{t}+P_{t} \exp [(\mu-k)(\tau-t)] \mid P_{t}=x\right] .
\end{gathered}
$$

Consequently, we have to determine:

$$
\sup _{\tau \in \mathrm{J}_{t, \bar{T}}} E\left[c\left(e^{-a t}-e^{-a \tau}\right)+P_{t} \exp [(\mu-k)(\tau-t)] \mid P_{t}=x\right] .
$$

In particular, we have to search for the value $\tau_{t}^{*}$ for which the maximum

$$
\sup _{\tau \in J_{t, \bar{T}}} E\left[-c e^{-a \tau}+x \exp [(\mu-k)(\tau-t)] \mid P_{t}=x\right],
$$

is achieved. This problem is the dynamic version of the determination of $T^{*}$ presented in Section 2. 
Introduce the function $f_{t, x}$ defined by:

$$
f_{t, x}(\theta)=-c e^{-a(t+\theta)}+x \exp [-b \theta] \text { with } b=k-\mu .
$$

Case 1. The asset value $x\left(=P_{t}\right)$ is smaller than $\frac{a c}{b} e^{-a t} e^{-(a-b)(\bar{T}-t)}$.

Then, the optimal time $\tau_{t}^{*}$ corresponds to the maturity $\bar{T}$ and

$$
\mathrm{V}(x, t)=c\left(e^{-a t}-e^{-a \bar{T}}\right)+x \exp [-b(\bar{T}-t)]
$$

Case 2: The asset value $x$ lies between the two values $\frac{a c}{b} e^{-a t} e^{-(a-b)(\bar{T}-t)}$ and $\frac{a c}{b} e^{-a t}$.

Then, the optimal time $\tau_{t}^{*}$ is equal to $\left(t+\theta^{*}\right)$, where $\theta^{*}$ is the solution of the following equation:

$$
\frac{\partial f_{t, x}}{\partial \theta}(\theta)=0
$$

Then, from Equation (25), we deduce:

$$
\theta^{*}=\frac{1}{a-b} \ln \left(\frac{a c e^{-a t}}{b x}\right) .
$$

Therefore, knowing that $P_{t}=x$ at time $t$, the time $\tau_{t}^{*}$ is deterministic. Then, the value function $\mathrm{V}(x, t)$ is given by:

$$
\mathrm{V}(x, t)=c\left(e^{-a t}-e^{-a \tau_{t}^{*}}\right)+x \exp \left[-b\left(\tau_{t}^{*}-t\right)\right]
$$

from which, we get:

$$
\mathrm{V}(x, t)=c e^{-a t}+\left(\frac{a-b}{a}\right)\left(\frac{a c}{b} e^{-a t}\right)^{\left(-\frac{b}{a-b}\right)} x^{\left(\frac{a}{a-b}\right)}
$$

Case 3: The asset value $x$ is higher than $\frac{a c}{b} e^{-a t}$.

Then, the optimal time $\tau_{t}^{*}$ corresponds to the present time $t$, and

$$
\mathrm{V}(x, t)=x \text {. }
$$

Consequently, from the three previous cases, we deduce:

$$
\mathrm{V}(x, t)=\left\{\begin{array}{c}
c\left(e^{-a t}-e^{-a \bar{T}}\right)+x e^{-b(\bar{T}-t)}, \text { if } x \leq \frac{a c}{b} e^{-a t} e^{-(a-b)(\bar{T}-t)} \\
c e^{-a t}+\left(\frac{a-b}{a}\right)\left(\frac{a c}{b} e^{-a t}\right)^{\left(-\frac{b}{a-b}\right)} x^{\left(\frac{a}{a-b}\right)}, \text { if } \frac{a c}{b} e^{-a t} e^{-(a-b)(\bar{T}-t)}<x<\frac{a c}{b} e^{-a t} \\
x, \text { if } x \geq \frac{a c}{b} e^{-a t}
\end{array}\right.
$$


Figure 14 illustrates the function value $\mathrm{V}(x, t)$ (for $t=\bar{T} / 2$ and parameter values of the basic numerical example). Note that $\frac{a c}{b} e^{-a t} \simeq 65.5$ )

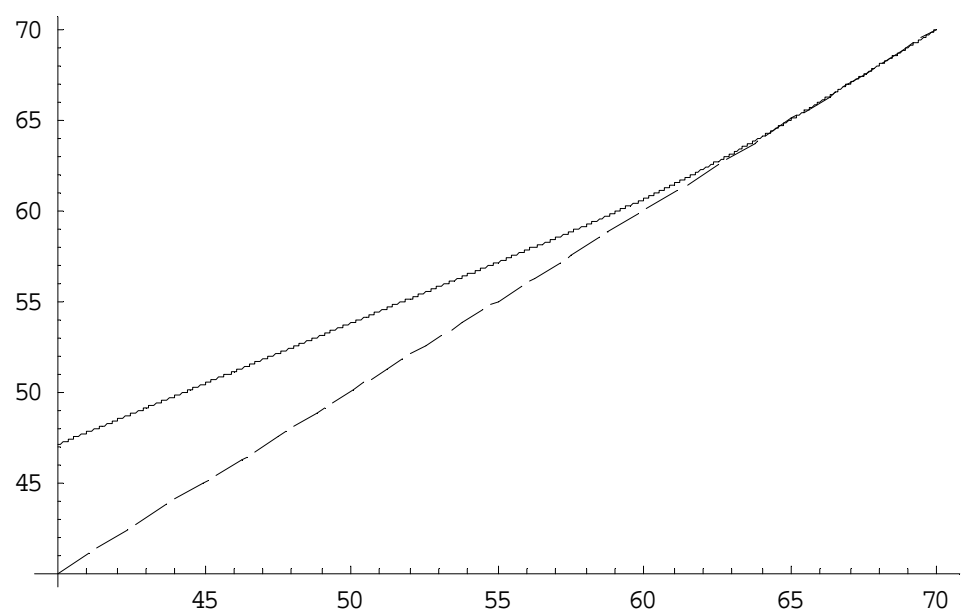

Fig. 14 Value function $V$

Finally, the American optimal time $T^{* * *}$ is determined by:

$$
T^{* * *}=\inf \left\{t \in[0, \bar{T}] \mid \mathrm{V}\left(P_{t}, t\right)=P_{t}\right\} .
$$

Therefore, we can check that $\mathrm{V}\left(P_{t}, t\right)=P_{t}$ if and only if $P_{t} \geq \frac{F C F_{0}}{(k-\mu)} e^{-(k-g) t}$. Thus, we have:

$$
T^{* * *}=\inf \left\{t \in[0, \bar{T}] \mid P_{t} \geq \frac{F C F_{0}}{(k-\mu)} e^{-(k-g) t}\right\} .
$$

Using standard results about the first time $T_{y}^{(m)}$ at which a Brownian motion with drift $W^{(m)}$ reaches a given level $y$, we can derive the pdf and cdf of $T^{* * *}$. Indeed, the condition $P_{t} \geq \frac{F C F_{0}}{(k-\mu)} e^{-(k-g) t}$ is equivalent to

$$
\begin{aligned}
P_{0} \exp \left[\left(\mu-k-1 / 2 \sigma^{2}\right) t+\sigma W_{t}\right] & \geq \frac{F C F_{0}}{(k-\mu)} e^{-(k-g) t}, \\
\left(\mu-g-1 / 2 \sigma^{2}\right) t+\sigma W_{t} & \geq \ln \left[\frac{F C F_{0}}{P_{0}(k-\mu)}\right], \\
\left(\frac{\mu-g}{\sigma}-1 / 2 \sigma\right) t+W_{t} & \geq \frac{1}{\sigma} \ln \left[\frac{F C F_{0}}{P_{0}(k-\mu)}\right] .
\end{aligned}
$$

Setting $m=\frac{\mu-g}{\sigma}-1 / 2 \sigma$ and $y=\frac{1}{\sigma} \ln \left[\frac{F C F_{0}}{P_{0}(k-\mu)}\right]$, the cdf of the random $T_{y}$ is given by:

- For the case $\frac{F C F_{0}}{P_{0}(k-\mu)} \leq 1$, we have:

$$
T^{* * *}=0
$$

- For the case $\frac{F C F_{0}}{P_{0}(k-\mu)}>1$, we have:

$$
P\left[T_{y} \leq t\right]=1-G(m, y, t)
$$


Thus, since $P\left[T^{* * *} \leq t\right]=P\left[T_{y}^{(m)} \leq t\right]$ for any $t<\bar{T}$, we deduce

$$
\begin{aligned}
P\left[T^{* * *} \leq t\right] & =\frac{1}{2} \operatorname{Erfc}\left(\frac{y}{\sqrt{2 t}}-m \frac{\sqrt{t}}{\sqrt{2}}\right)+\frac{1}{2} e^{2 m y} \operatorname{Erfc}\left(\frac{y}{\sqrt{2 t}}+m \frac{\sqrt{t}}{\sqrt{2}}\right), \\
& \text { and } \\
P\left[T^{* * *}=\bar{T}\right] & =P\left[T_{y}^{(m)}=\bar{T}\right]+P\left[T_{y}^{(m)}>\bar{T}\right]=0+P\left[T_{y}^{(m)}>\bar{T}\right]= \\
& =1-\frac{1}{2} \operatorname{Erfc}\left(\frac{y}{\sqrt{2 \bar{T}}}-m \frac{\sqrt{\bar{T}}}{\sqrt{2}}\right)-\frac{1}{2} e^{2 m y} \operatorname{Erfc}\left(\frac{y}{\sqrt{2 \bar{T}}}+m \frac{\sqrt{\bar{T}}}{\sqrt{2}}\right) .
\end{aligned}
$$

B.4. Computation of the optimal value $V_{T^{*+3 t}}$

At time $T^{* * *}$, we have $P_{T^{* * * *}}=\frac{F C F_{0}}{(k-\mu)} e^{-(k-g) T^{* * * *}}$ if $T^{* * *}<\bar{T}$. Thus, the portfolio value $V_{T^{* * *}}$ is given by:

$$
\begin{gathered}
V_{T^{* * *}}=\frac{F C F_{0}}{(k-g)}\left(1+e^{-(k-g) T^{* * * *}} \frac{(\mu-g)}{(k-\mu)}\right), \text { if } T^{* * *}<\bar{T}, \\
\text { and } \\
V_{T^{* * * *}}=\frac{F C F_{0}}{(k-g)}\left(1-e^{-(k-g) \bar{T}}\right)+P_{0} \exp \left[\left(\mu-k-1 / 2 \sigma^{2}\right) \bar{T}+\sigma W_{\bar{T}}\right], \\
\text { if } T^{* * * *}=\bar{T} .
\end{gathered}
$$

\section{B.5. Computation of the cdf $F_{V_{T^{m+n}}}$}

We have:

$$
F_{V_{T^{* * *}}}(v)=P\left[V_{T^{* * *}} \leq v \cap T^{* * *}<\bar{T}\right]+P\left[V_{T^{* * * *}} \leq v \cap T^{* * *}=\bar{T}\right]
$$

i) Computation of $P\left[V_{T^{* * *}} \leq v \cap T^{* * *}<\bar{T}\right]$ :

Setting $g(v)$

$$
\begin{gathered}
P\left[V_{T^{* * *}} \leq v \cap T^{* * *}<\bar{T}\right] \\
=P\left[\frac{F C F_{0}}{(k-g)}\left(1+e^{-(k-g) T^{* * *}} \frac{(\mu-g)}{(k-\mu)}\right) \leq v \cap T^{* * *}<\bar{T}\right]
\end{gathered}
$$

$$
g(v)=\frac{1}{k-g} \log \left[\frac{\mu-g}{k-\mu} \times \frac{\frac{F C F_{0}}{k-g}}{v-\frac{F C F_{0}}{k-g}}\right],
$$

We have

$$
g(v) \leq \bar{T} \Longleftrightarrow v \geq \frac{F C F_{0}}{k-g}\left[1+\frac{\mu-g}{k-\mu} e^{-(k-g) \bar{T}}\right]
$$


Therefore,

$$
\begin{aligned}
P\left[V_{T^{* * *}} \leq v \cap T^{* * *}<\bar{T}\right] & =P\left[T^{* * *} \geq g(v) \cap T^{* * *}<\bar{T}\right] \\
& =\left\{\begin{array}{c}
0, \text { if } g(v)>\bar{T} \\
P\left[g(v) \leq T^{* * *} \leq \bar{T}\right], \text { if } g(v) \leq \bar{T}
\end{array}\right.
\end{aligned}
$$

But $P\left[T^{* * *} \leq g(v)\right]=0$, if $g(v)<0$, that is if $v>\frac{F C F_{0}}{k-g}\left[1+\frac{\mu-g}{k-\mu}\right]$.

ii) Computation of $P\left[V_{T^{* * *}} \leq v \cap T^{* * *}=\bar{T}\right]$

$$
\begin{gathered}
P\left[V_{T^{* * * *}} \leq v \cap T^{* * *}=\bar{T}\right]= \\
P\left[\frac{F C F_{0}}{(k-g)}\left(1-e^{-(k-g) \bar{T}}\right)+P_{0} \exp \left[\left(\mu-k-1 / 2 \sigma^{2}\right) \bar{T}+\sigma W_{\bar{T}}\right] \leq v\right] . \\
\cap \sup _{0 \leq s \leq \bar{T}} W_{s}^{(m)} \leq y
\end{gathered}
$$

Recall that, for a geometric Brownian with drift $W^{(m)}$, the joint distribution of its maximum on $[0, \bar{T}]$ and its value at time $\bar{T}$ is given by (see Borodin and Salminen (2002), p. 251),

$$
P\left[\operatorname{Sup}_{0 \leq t \leq \bar{T}} W_{t}^{(m)} \geq y, W_{\bar{T}}^{(m)} \in d z\right]=\frac{1}{\sqrt{2 \pi \bar{T}}} e^{m z-m^{2} \bar{T} / 2-(|z-y|+y)^{2} / 2 \bar{T}} d z .
$$

Since

$$
P\left[\operatorname{Sup}_{0 \leq t \leq \bar{T}} W_{t}^{(m)}<y, W_{\bar{T}}^{(m)} \in d z\right]=P\left[W_{\bar{T}}^{(m)} \in d z\right]-\frac{1}{\sqrt{2 \pi \bar{T}}} e^{m z-m^{2} \bar{T} / 2-(|z-y|+y)^{2} / 2 \bar{T}} d z,
$$

finally we get:

$$
P\left[T^{* * *}=\bar{T}, W_{\bar{T}}^{(m)} \in d z\right]=P\left[W_{\bar{T}}^{(m)} \in d z\right]-\frac{1}{\sqrt{2 \pi \bar{T}}} e^{m z-m^{2} \bar{T} / 2-(|z-y|+y)^{2} / 2 \bar{T}} d z .
$$

Consequently, for $v>\frac{F C F_{0}}{k-g}\left(1-e^{-(k-g) \bar{T}}\right)$, we have:

$$
\begin{gathered}
P\left[V_{T^{* * *}} \leq v \cap T^{* * *}=\bar{T}\right] \\
=P\left[T^{* * *}=\bar{T}, W_{\bar{T}}^{(m)}<\frac{1}{\sigma} \ln \left[\frac{v-\frac{F C F_{0}}{(k-g)}\left(1-e^{-(k-g) \bar{T}}\right)}{P_{0}}\right]+\frac{(k-g)}{\sigma} \bar{T}\right] .
\end{gathered}
$$

Recall that $y=\frac{1}{\sigma} \ln \left[\frac{F C F_{0}}{P_{0}(k-\mu)}\right]$ and $m=\frac{\mu-g}{\sigma}-1 / 2 \sigma$. Denote also

$$
z(v)=\frac{1}{\sigma} \ln \left[\frac{v-\frac{F C F_{0}}{(k-g)}\left(1-e^{-(k-g) \bar{T}}\right)}{P_{0}}\right]+\frac{(k-g)}{\sigma} \bar{T} .
$$


Then,

$$
\begin{gathered}
P\left[V_{T^{* * *}} \leq v \cap T^{* * *}=\bar{T}\right] \\
=\int_{-\infty}^{z(v)}\left(P\left[W_{\bar{T}}^{(m)} \in d z\right]-\frac{1}{\sqrt{2 \pi \bar{T}}} e^{m z-m^{2} \bar{T} / 2-(|z-y|+y)^{2} / 2 \bar{T}}\right) d z,
\end{gathered}
$$

with

$$
\int_{-\infty}^{z(v)}\left(P\left[W_{\bar{T}}^{(m)} \in d z\right]\right) d z=P\left[W_{\bar{T}}^{(m)} \leq z(v)\right]=N\left(\frac{z(v)-m \bar{T}}{\sqrt{\bar{T}}}\right)
$$

To determine $\frac{1}{\sqrt{2 \pi \bar{T}}} \int_{-\infty}^{z} e^{m z-m^{2} \bar{T} / 2-(|z-y|+y)^{2} / 2 \bar{T}} d z$, we have to compare $y$ and $z(v)$, according to the values of $v$ :

$$
z(v) \leq y \Longleftrightarrow v \leq \frac{F C F_{0}}{(k-g)}\left[1+e^{-(k-g) \bar{T}} \frac{\mu-g}{k-\mu}\right]
$$

- $\quad$ If $z(v) \leq y$

$$
\begin{aligned}
& \frac{1}{\sqrt{2 \pi \bar{T}}} \int_{-\infty}^{z(v)} e^{m u-m^{2} \bar{T} / 2-(|u-y|+y)^{2} / 2 \bar{T}} d u=\frac{1}{\sqrt{2 \pi \bar{T}}} \int_{-\infty}^{z} e^{m u-m^{2} \bar{T} / 2-(2 y-u)^{2} / 2 \bar{T}} d u \\
& =e^{2 y m} \frac{1}{\sqrt{2 \pi \bar{T}}} \int_{-\infty}^{z(v)} e^{-\frac{1}{2 \bar{T}}[u-(m \bar{T}+2 y)]^{2}} d u=e^{2 y m} N\left(\frac{z(v)-(m \bar{T}+2 y)}{\sqrt{\bar{T}}}\right) .
\end{aligned}
$$

- If $z(v)>y$

$$
\begin{gathered}
\frac{1}{\sqrt{2 \pi \bar{T}}} \int_{-\infty}^{z(v)} e^{m u-m^{2} \bar{T} / 2-(|u-y|+y)^{2} / 2 \bar{T}} d u \\
=\frac{1}{\sqrt{2 \pi \bar{T}}} \int_{-\infty}^{y} e^{m u-m^{2} \bar{T} / 2-(2 y-u)^{2} / 2 \bar{T}} d u+\frac{1}{\sqrt{2 \pi \bar{T}}} \int_{y}^{z(v)} e^{m u-m^{2} \bar{T} / 2-u^{2} / 2 \bar{T}} d u, \\
=e^{2 y m} N\left(\frac{-y-m \bar{T}}{\sqrt{2 \pi \bar{T}}}\right)+\left[N\left(\frac{z(v)-m \bar{T}}{\sqrt{2 \pi \bar{T}}}\right)-N\left(\frac{y-m \bar{T}}{\sqrt{2 \pi \bar{T}}}\right)\right] .
\end{gathered}
$$

Note also that we always have:

$$
\frac{F C F_{0}}{k-g}\left(1-e^{-(k-g) \bar{T}}\right) \leq \frac{F C F_{0}}{k-g}\left(1+\frac{\mu-g}{k-\mu} e^{-(k-g) \bar{T}}\right) \leq \frac{F C F_{0}}{k-g}\left(1+\frac{\mu-g}{k-\mu}\right) .
$$

Consequently, the cdf of $V_{T^{* * *}}$ is defined by:

- If $v \leq \frac{F C F_{0}}{k-g}\left(1-e^{-(k-g) \bar{T}}\right)$

$$
F_{V_{T^{* * *}}}(v)=0
$$




$$
\begin{aligned}
& \text { - If } \frac{F C F_{0}}{k-g}\left(1-e^{-(k-g) \bar{T}}\right)<v<\left(\frac{F C F_{0}}{k-g}\right)\left(1+\frac{\mu-g}{k-\mu} e^{-(k-g) \bar{T}}\right), \\
& \qquad F_{V_{T^{* * *}}}(v)=N\left(\frac{z(v)-m \bar{T}}{\sqrt{\bar{T}}}\right)-e^{2 y m} N\left(\frac{z(v)-(m \bar{T}+2 y)}{\sqrt{\bar{T}}}\right) \\
& \text { - If }\left(\frac{F C F_{0}}{k-g}\right)\left(1+\frac{\mu-g}{k-\mu} e^{-(k-g) \bar{T}}\right) \leq v \leq \frac{F C F_{0}}{k-g}\left(1+\frac{\mu-g}{k-\mu}\right), \\
& \qquad F_{V_{T^{* * *}}}(v)=G(m, y, g(v))-G(m, y, \bar{T}) \\
& +N\left(\frac{z(v)-m \bar{T}}{\sqrt{\bar{T}}}\right)-e^{2 y m} N\left(\frac{-y-m \bar{T}}{\sqrt{\bar{T}}}\right)-\left[N\left(\frac{z(v)-m \bar{T}}{\sqrt{2 \bar{T}}}\right)-N\left(\frac{y-m \bar{T}}{\sqrt{2 \bar{T}}}\right)\right] \\
& \text { - If } \frac{F C F_{0}}{k-g}\left(1+\frac{\mu-g}{k-\mu}\right)<v \quad F_{V_{T^{* * *}}}(v)=1-G(m, y, \bar{T}) \\
& +N\left(\frac{z(v)-m \bar{T}}{\sqrt{\bar{T}}}\right)-e^{2 y m} N\left(\frac{-y-m \bar{T}}{\sqrt{\bar{T}}}\right)-\left[N\left(\frac{z(v)-m \bar{T}}{\sqrt{2 \bar{T}}}\right)-N\left(\frac{y-m \bar{T}}{\sqrt{2 \bar{T}}}\right)\right] .
\end{aligned}
$$

\section{Acknowledgments}

We gratefully acknowledge Shaun Bond (discussant) and participants at the MITCambridge-Maastricht Conference, 2007.

\section{Notes}

1. These optimization problems are specific to real estate investments and differ from standard financial portfolio management problems (see Karatzas and Shreve, 2001, or Prigent, 2007). First, the asset is not liquid (not divisible). Second, the control variable is the time to sell and not the usual financial portfolio weights (see Oksendal 2007, for a related problem about optimal time to invest in a project with an infinite horizon).

2. This assumption allows explicit solutions for the probability distributions of the optimal times to sell and of the optimal portfolio values. The introduction of stochastic rates would lead to only simulated solutions.

3. The two other cases $\mu<g<k$ and $\mu<k<g$ could be analyzed in the same way.

4. This is the continuous-time version of the solution of Baroni et al. (2007b).

5. We can also examine how the solution depends on the index value $P_{0}$. For example, proportional transaction costs imply a reduction of $P_{0}$. For instance, for the case 2, a tax of 5\% leads to an optimal time to sell $T^{*}$ equal to 17.39 years, instead of 16.11 years when there is no transaction cost. With a $10 \%$ tax, the solution becomes 18.74 years. This is in line with the empirical results showing that high transaction costs imply longer holding periods (see for example Collet et al., 2003).

6. See Elliott and Kopp (1999, p. 193). 


\section{References}

[1] Baroni, M., Barthélémy, F., and M. Mokrane, (2007a): "Monte Carlo Simulations versus DCF in Real Estate Portfolio Valuation", Property Management, 25(5), 462486.

[2] Baroni, M., Barthélémy, F., and M. Mokrane, (2007b): “Optimal Holding Period for a Real Estate Portfolio", Journal of Property Investment and Finance, 25(6), 603625.

[3] Bond, S.A., Hwang, S., Lin, Z. and K.D. Vandell, (2007): Marketing Period Risk in a Portfolio Context: Theory and Empirical Estimates from the UK Commercial Real Estate Market, Journal of Real Estate Finance and Economics, Vol. 34, 447461.

[4] Borodin, A. N., and P. Salminen, (2002): Handbook of Brownian Motion (Facts and Formulae), Birkhäuser.

[5] Brown, R.J., (2004): "Risk and private Real Estate Investments", Journal of Real Estate Portfolio Management, Vol. 10, 113.

[6] Brown, R.J., and T. Geurts, (2005): "Private Investor Holding Period", Journal of Real Estate Portfolio Management, Vol. 11, 93-104.

[7] Collett, D, Lizieri, C., and C.W.R. Ward, (2003), Timing and the Holding Periods of Institutional Real Estate. Real Estate Economics, 31, 205-222.

[8] Elliott, R.J., and Kopp, P.E., (1999): Mathematics of Financial Markets, Springer Finance series, Springer.

[9] Fisher, J., and M. Young, (2000): "Institutional Property Tenure: evidence from the NCREIF database", Journal of Real Estate Portfolio Management, Vol. 6, 327338.

[10] Gau, G., and K. Wang, (1994): "The Tax-induced Holding Periods and Real Estate Investors: Theory and Empirical Evidence", Journal of Real Estate Finance and Economics, Vol. 8, 71-86.

[11] Geltner, D. M., and N.G. Miller, (2007): Commercial Real Estate Analysis and Investments, Cengage/South-Western, Cincinnati.

[12] Hendershott, P., and D. Ling, (1984): "Prospective Changes in Tax Law and the Value of Depreciable Real Estate”, AREUEA Journal, Vol. 12, 297-317.

[13] Karatzas, I., and S. Shreve, (2001): Methods of Mathematical Finance, SpringerVerlag.

[14] Oksendal, B., (2007): Stochastic Differential Equations: An introduction with applications, 6th ed. 2003. (Corr. 4th), Springer-Verlag.

[15] Prigent, J-L., (2007): Portfolio Optimization and Performance Analysis, Chapman \& Hall, Florida.

[16] Rowley, A., V. Gibson, and C. Ward, (1996): "Quality of Urban Design: a Study of the Involvement of Private Property Decision-Makers in Urban Design", Royal Institution of Chartered Institution of Chartered Surveyors, London. 